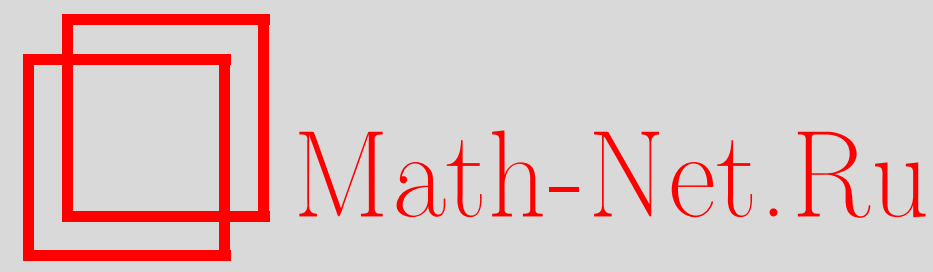

И. К. Лифанов, Л. Н. Полтавский, Псевдоразностные операторы и равномерная сходимость разностных отношений, Матем. сб., 2002, том 193, номер 2, 53-80

DOI: https://doi.org/10.4213/sm627

Использование Общероссийского математического портала Math-Net.Ru подразумевает, что вы прочитали и согласны с пользовательским соглашением http://www . mathnet.ru/rus/agreement

Параметры загрузки:

IP: 3.91 .87 .62

26 апреля 2023 г., 16:23:57 


\title{
Псевдоразностные операторы и равномерная сходимость разностных отношений
}

\begin{abstract}
В работе вводится понятие псевдоразностного оператора (п.р.о.). Исследованы свойства некоторого класса п.р.о. в пространствах дробных отношений. Получена локальная теорема о равномерной сходимости разностных отношений любого порядка приближенного решения. В частности, на основе численного метода доказана локальная бесконечная дифференцируемость точного решения операторных уравнений эллиптического типа при локальной бесконечной диффференцируемости правой части. Приведены примеры, связанные с приложениями.

Библиография: 3 названия.
\end{abstract}

\section{Введение}

Для численного решения задач обтекания несущих поверхностей идеальной несжимаемой жидкостью в аэродинамике большое применение нашел метод дискретных вихрей. В работах [1], [2] введено и исследовано понятие пространств дробных отношений. Это дало возможность обосновать метод вихревых рамок для некоторых прикладных задач. В настояшей работе вводится понятие псевдоразностного оператора (п.р.о.). Исследованы свойства некоторого класса п.р.о. в пространствах дробных отношений. На основе описанных выше результатов получена локальная теорема о равномерной сходимости разностных отношений любого порядка. В частности, исходя из численного метода доказана локальная бесконечная дифференцируемость решения операторных уравнений эллиптического типа при локальной бесконечной дифференцируемости правой части. Приведены примеры.

\section{§1. Дискретное преобразование Фурье}

Напомним некоторые определения из [1]. В евклидовом пространстве $\mathbb{R}_{n}$ возьмем какую-нибудь декартову систему координат $O X_{1}, \ldots, X_{n}$. Каждую ось $X_{k}$ разобьем с шагом $h_{k}$ точками $x_{k}^{i}=h_{k}(i-1 / 2), i \in \mathbb{Z}$. Тогда все пространство $\mathbb{R}_{n}$ разобьется на ячейки

$$
D\left(k_{1}, \ldots, k_{n}, h_{1}, \ldots, h_{n}\right)=\prod_{j=1}^{n}\left(h_{j}\left(k_{j}-\frac{1}{2}\right), h_{j}\left(k_{j}+\frac{1}{2}\right)\right), \quad k_{j} \in \mathbb{Z}
$$

символ $\prod$ здесь понимается как декартово произведение интервалов $h_{j}\left(k_{j}-1 / 2\right)<$ $x_{j}<h_{j}\left(k_{j}+1 / 2\right)$. В дальнейшем будем обозначать $D\left(k_{1}, \ldots, k_{n}, h_{1}, \ldots, h_{n}\right)=$ $D(k, h)$, где $k=\left(k_{1}, \ldots, k_{n}\right), h=\left(h_{1}, \ldots, h_{n}\right)$. 
ОПРеДЕЛЕниЕ 1.1. Разбиение пространства $\mathbb{R}_{n}$ на ячейки $D(k, h)$ будем называть каноническим разбиением, а множество точек $M\left(k_{1} h_{1}, \ldots, k_{n} h_{n}\right)$ - канонической сеткой пространства $\mathbb{R}_{n}$; функции $a\left(k_{1}, \ldots, k_{n}\right)=a(k)$, определенные в узлах $M\left(k_{1} h_{1}, \ldots, k_{n} h_{n}\right)$ канонической сетки, будем называть сеточными.

Обозначим через $M_{h}$ множество ступенчатых комплекснозначных функций $a(x, h)$, которые на каждой ячейке канонического разбиения $D(k, h)$ принимают постоянное значение; множество комплекснозначных сеточных функций $a(k)$ обозначим через $M_{k}$.

ОПРЕДЕЛЕНИЕ 1.2. Стандартным отображением множества $M_{h}$ на множество $M_{k}$ будем называть оператор $\pi$, определенный соотношением:

$$
\pi a(x, h)=a(k), \text { где } a(x, h)=a(k) \text { при } x \in D(k, h) .
$$

Рассмотрим каноническое разбиение $\mathbb{R}_{n}$ на ячейки $D(k, h)$ с векторным шагом $h=\left(h_{1}, \ldots, h_{n}\right)$. Каждой локально суммируемой функции $f(x)$ поставим в соответствие сеточную функцию $a(k)$ по правилу:

$$
a(k)=\frac{1}{\Omega_{h}} \int_{D(k, h)} f(x) d x
$$

где $\Omega_{h}=h_{1} \cdots h_{n}$. Далее, сеточной функции $a(k)$ поставим в соответствие ступенчатую функцию $a(x, h) \in M_{h}$ такую, что $a(k)=\pi a(x, h)$.

ОПРЕДЕЛЕНИЕ 1.3. Оператор, ставящий в соответствие локально суммируемой функции $f(x)$ функцию $a(x, h) \in M_{h}$ по описанному вьшше правилу, будем называть интегральным проектором и обозначать через $\prod_{h}$, т.е. $\prod_{h} f(x)=a(x, h)$.

ОПРЕДЕЛЕНИЕ 1.4. Пространство ступенчатых функиий $M_{h}^{\infty}$ по определению состоит из функций вида $\prod_{h} \varphi(x)$, где $\varphi(x) \in C_{0}^{\infty}\left(\mathbb{R}_{n}\right)$ - финитные бесконечно дифференцируемые функции.

Обозначим через $\mathbb{Z}_{n}$ теоретико-множественное произведение $n$ экземпляров множества целых чисел $\mathbb{Z}$, а через $\mathbb{Z}_{n}(N)$ - теоретико-множественное произведение $n$ экземпляров множества целых чисел, элементы которого $k$ удовлетворяют неравенству $-N \leqslant k \leqslant N$.

Пусть функция $a(x, h) \in M_{h}$ принадлежит также $L_{p}\left(\mathbb{R}_{n}\right), 1 \leqslant p \leqslant 2$, т.е. для нее имеет место $\int_{\mathbb{R}_{n}}|a(x, h)|^{p} d x \leqslant C$, что эквивалентно выполнению условия

$$
\sum_{k \in \mathbb{Z}_{n}}|\pi a(x, h)|^{p} \leqslant \frac{C}{\Omega_{h}}
$$


ОПРЕДЕЛЕНИЕ 1.5. Дискретныц ступенчатым преобразованием Фурье $F_{h} a(x, h)$ функции $a(x, h) \in M_{h}, a(x, h) \in L_{p}\left(\mathbb{R}_{n}\right), 1 \leqslant p \leqslant 2$, будем называть функцию, определенную соотношением

$$
F_{h} a(x, h)=\widehat{a}_{h}(\varphi)=\Omega_{h} \cdot \sum_{k \in \mathbb{Z}_{n}} a(k) e^{i(k \varphi, h)},
$$

где $k \varphi=\sum_{i=1}^{n} k_{i} \varphi_{i},(k \varphi, h)=\sum_{i=1}^{n} k_{i} \varphi_{i} h_{i}, a(k)=\pi a(x, h), k_{i} \in \mathbb{Z}, \Omega_{h}=$ $\prod_{j=1}^{n} h_{j}$.

Правая часть в (1.1) понимается следуюшим образом:

$$
\widehat{a}_{h}(\varphi)=\lim _{N \rightarrow+\infty} \Omega_{h} \sum_{k \in \mathbb{Z}_{n}(N)} a(k) e^{i(k \varphi, h)} .
$$

Функция $\widehat{a}_{h}(\varphi)$, определенная соотношением (1.1), периодична по каждому аргументу $\varphi_{i}$ с периодом $2 \pi / h_{i}$, кроме того,

$$
\widehat{a}_{h}(\varphi) \in L_{p_{1}}\left[D_{h}\right], \quad D_{h}=\prod_{i=1}^{n}\left[-\frac{\pi}{h_{i}} \leqslant \varphi_{i} \leqslant \frac{\pi}{h_{i}}\right], \quad \frac{1}{p}+\frac{1}{p_{1}}=1
$$

(см. [3]). Значение $a(k)$ ступенчатой функции $a(x, h)$ на ячейке $D(k, h)$ восстанавливается по формуле

$$
a(k)=\frac{1}{(2 \pi)^{n}} \int_{D_{h}} \widehat{a}_{h}(\varphi) e^{i(k \varphi, h)} d \varphi
$$

ОПРЕДЕЛЕНИЕ 1.6. Дискретныцм сеточныцм преобразованием Фурье $F_{c} a(k)$ сеточной функции $a(k) \in l_{p}, 1 \leqslant p \leqslant 2$, будем называть функцию, определенную соотношением

$$
F_{c} a(k)=\widehat{a}_{c}(\varphi)=\lim _{N \rightarrow \infty} \sum_{k \in \mathbb{Z}_{n}(N)} a(k) e^{i(k \varphi)}
$$

Значения сеточной функции $a(k)$ восстанавливаются по формуле

$$
a(k)=\frac{1}{(2 \pi)^{n}} \int_{U} e^{-i(k \varphi)} \widehat{a}_{c}(\varphi) d \varphi,
$$

где $U=\prod_{j=1}^{n}\left[-\pi \leqslant \varphi_{j} \leqslant \pi\right]$.

Для дискретного ступенчатого и сеточного преобразования Фурье имеет место аналог равенства Парсеваля:

$$
\begin{aligned}
\int_{U} \widehat{a}_{c}(\varphi) \overline{\widehat{b}_{c}(\varphi)} d \varphi & =(2 \pi)^{n} \sum_{k} a(k) \overline{b(k)}=\frac{(2 \pi)^{n}}{\Omega_{h}} \int_{\mathbb{R}_{n}} a(x, h) \overline{b(x, h)} d x, \\
\int_{D_{h}} \widehat{a}_{h}(\varphi) \overline{\widehat{b}_{h}(\varphi)} d \varphi & =(2 \pi)^{n} \Omega_{h} \sum_{k} a(k) \overline{b(k)}=(2 \pi)^{n} \int_{\mathbb{R}_{n}} a(x, h) \overline{b(x, h)} d x .
\end{aligned}
$$


ПРЕДЛОЖЕНИЕ 1.1. Если функция $a(x, h) \in M_{h}^{\infty}$, то при $|h| \leqslant h_{0}$ въполняется неравенство

$$
\left|\widehat{a}_{h}(\varphi)\right| \leqslant \frac{C_{N}}{\left(1+\sum_{i=1}^{n}\left|\frac{1}{h_{i}} \sin \frac{\varphi_{i} h_{i}}{2}\right|\right)^{N}},
$$

где $C_{N}$ не зависит от $h=\left(h_{1}, \ldots, h_{n}\right)$.

ДоКАЗАТЕЛЬСтво. Легко проверить, что имеет место тождество

$$
e^{i(k \varphi, h)}=\frac{\left[\Delta_{p}^{N}+(-1)^{N}\right] e^{i(k \varphi, h)}}{(-1)^{N} 4^{N}\left(\sum_{i=1}^{n} \frac{1}{h_{i}^{2}} \sin ^{2} \frac{\varphi_{i} h_{i}}{2}\right)^{N}+(-1)^{N}},
$$

где $\Delta_{p}$ - разностный оператор Лапласа.

Используя (1.3) и преобразование Абеля, получаем

$$
\begin{aligned}
\widehat{a}_{h}(\varphi) & =\Omega_{h} \sum_{k \in \mathbb{Z}_{n}} a(k) e^{i(k \varphi, h)} \\
& =\Omega_{h} \sum_{k \in \mathbb{Z}_{n}} a(k) \frac{\left[\Delta_{p}^{N}+(-1)^{N}\right] e^{i(k \varphi, h)}}{(-1)^{N} 4^{N}\left(\sum_{i=1}^{n} \frac{1}{h_{i}^{2}} \sin ^{2} \frac{\varphi_{i} h_{i}}{2}\right)^{N}+(-1)^{N}} \\
& =\frac{\Omega_{h}}{(-1)^{N} 4^{N}\left(\sum_{i=1}^{n} \frac{1}{h_{i}^{2}} \sin ^{2} \frac{\varphi_{i} h_{i}}{2}\right)^{N}+(-1)^{N}} \sum_{k \in \mathbb{Z}_{n}} e^{i(k \varphi, h)}\left[\Delta_{p}^{N}+(-1)^{N}\right] a(k),
\end{aligned}
$$

где $a(k)=\pi a(x, h)$.

Так как $a(x, h)=\prod_{h} \chi(x), \chi(x) \in C_{0}^{\infty}\left(\mathbb{R}_{n}\right)$, то $a(x, h) \in M(r, h) \forall r>0$ $(M(r, h)$ - пространство дробных отношений, см. [1]). Из теории вложения для пространств дробных отношений (см. [1]) следует оценка

$$
\left|\left[\Delta_{p}^{N}+(-1)^{N}\right] a(k)\right| \leqslant C(N) .
$$

С другой стороны, функция $a(x, h)$ финитна, поэтому $V(k)=\left[\Delta_{p}^{N}+(-1)^{N}\right] a(k)$ является финитной функцией. Следовательно, при $|h|<h_{0}$ ( $h_{0}$ фиксировано) будет выполняться неравенство

$$
\text { mes } \operatorname{supp} V(k) \leqslant C_{1}(N)
$$

Из (1.4)-(1.6) следует оценка

$$
\left|\widehat{a}_{h}(\varphi)\right| \leqslant \frac{A_{N}}{1+\left(\sum_{i=1}^{n} \frac{1}{h_{i}^{2}} \sin ^{2} \frac{\varphi_{i} h_{i}}{2}\right)^{N}} \leqslant \frac{C_{N}}{\left(1+\sum_{i=1}^{n} \frac{1}{h_{i}^{2}} \sin ^{2} \frac{\varphi_{i} h_{i}}{2}\right)^{N}} .
$$

Из последнего неравенства следует утверждение предложения 1.1.

Каждой функции $f(x) \in C\left(\mathbb{R}_{n}\right)$ можно поставить в соответствие сеточную функцию $a(k)$ по правилу: $a\left(k_{1}, \ldots, k_{n}\right)=f\left(k_{1} h_{1}, \ldots, k_{n} h_{n}\right)$. Сеточной функции $a(k)$ поставим в соответствие ступенчатую функцию $a(x, h)$ такую, что $\pi a(x, h)=a(k)$. 
ОПРЕДЕЛЕНИЕ 1.7. Оператор, ставящий в соответствие функции $f(x) \in C\left(\mathbb{R}_{n}\right)$ ступенчатую функцию $a(x, h)$ по описанному выше правилу, будем называть точечным проектором и обозначать через $\prod_{h}^{*}$, т.е. $\prod_{h}^{*} f(x)=a(x, h)$.

ЗАмЕЧАнИЕ 1.1. Предложение 1.1 имеет место для функций $\prod_{h}^{*} \chi(x)$, где $\chi(x) \in C_{0}^{\infty}\left(\mathbb{R}_{n}\right)$.

ОПРЕДЕЛЕНИЕ 1.8. Псевдоразностным оператором (п.р.о.) называется оператор, отображающий класс функций $M_{h}^{\infty}$ в класс сеточных функций $M_{k}$ и определенный соотношением

$$
\pi A_{h}(x)=\frac{1}{(2 \pi)^{n}} \int_{U} \widehat{A}_{c}(\varphi, k, h) \widehat{a}_{c}(\varphi) e^{-i(k \varphi)} d \varphi
$$

где $\pi$ - стандартный оператор (см. определение 1.2$), \widehat{a}_{c}(\varphi)$ - сеточное преобразование Фурье функции $a(k) \in M_{h}^{\infty}$; функция $\widehat{A}_{c}(\varphi, k, h)$ называется символом п.р.о., периодична по каждому аргументу с периодом $T=2 \pi, \widehat{A}_{c}(\varphi, k, h) \in L(U)$.

В интеграле (1.7) сделаем замену $\varphi_{i}=t_{i} \cdot h_{i}, i=1,2, \ldots, n$. Далее получим

$$
\pi A_{h}(x)=\frac{1}{(2 \pi)^{n}} \int_{D_{h}} \widehat{A}_{c}(t h, k, h) \widehat{a}_{h}(t) e^{-i(k t, h)} d t
$$

где $\widehat{a}_{h}(t)$ - ступенчатое преобразование Фурье функции $a(x, h), \pi a(x, h)=a(k)$; функцию $\widehat{A}_{h}(t, k, h)=\widehat{A}_{c}(t h, k, h)$ будем назьвать ступенчатым символомп.р.о.

ОПРЕДЕЛЕНИЕ 1.9. Будем говорить, что семейство п.р.о. $A_{h}$ со ступенчатыми символами $\widehat{A}_{h}(\varphi, h)$ (символ не зависит от $k$ ) принадлежит классу $S_{h \alpha}$, если существует такое $h_{0}>0$, что при $|h| \leqslant h_{0}$ вьполняется неравенство

$$
\left|\widehat{A}_{h}(\varphi, h)\right| \leqslant C\left(1+\sum_{i=1}^{n} \frac{1}{h_{i}}\left|\sin \frac{\varphi_{i} h_{i}}{2}\right|\right)^{\alpha}
$$

где $C$ не зависит от $h=\left(h_{1}, \ldots, h_{n}\right)$.

ОПРЕДЕЛЕНИЕ 1.10. Будем говорить, что семейство п.р.о. $A_{h}$ с постояннњми ступенчатыми символами $\widehat{A}_{h}(\varphi, h)$ принадлежит классу $S_{h \alpha}^{\infty}$, если существует такое $h_{0}>0$, что при $|h| \leqslant h_{0}$ выполняется неравенство

$$
\left|\frac{\partial^{k}}{\partial \varphi_{1}^{k_{1}} \cdots \partial \varphi_{n}^{k_{n}}} \widehat{A}_{h}(\varphi, h)\right| \leqslant C_{k}\left(1+\sum_{i=1}^{n} \frac{1}{h_{i}}\left|\sin \frac{\varphi_{i} h_{i}}{2}\right|\right)^{\alpha-|k|}
$$

$\varphi \in D_{h}$, причем функция $\frac{\partial^{k}}{\partial \varphi_{1}^{k_{1}} \cdots \partial \varphi_{n}^{k_{n}}} \widehat{A}_{h}(\varphi, h)$ непрерывна для любого $\varphi \in \mathbb{R}_{n}$, $|k|=k_{1}+\cdots+k_{n}$. 
ОПРЕДЕЛЕНИЕ 1.11. Будем говорить, что семейство п.р.о. $A_{h}$ с переменными ступенчатыми символами $\widehat{A}_{h}(\varphi, k, h)$ принадлежит классу $S_{h \alpha}$, если сушествует такое $h_{0}>0$, что при $|h| \leqslant h_{0}$ выполняется неравенство для любого $N$ :

$$
\left|\widehat{A}_{h}^{\prime}(\varphi, \psi, h)\right| \leqslant \frac{C_{N}\left(1+\sum_{i=1}^{n} \frac{1}{h_{i}}\left|\sin \frac{\varphi_{i} h_{i}}{2}\right|\right)^{\alpha}}{\left(1+\sum_{i=1}^{n} \frac{1}{h_{i}}\left|\sin \frac{\psi_{i} h_{i}}{2}\right|\right)^{N}}
$$

$\varphi, \psi \in D_{h}, C_{N}$ не зависит от $h=\left(h_{1}, \ldots, h_{n}\right)$, функция $\widehat{A}_{h}^{\prime}(\varphi, \psi, h)$ является ступенчатым преобразованием Фурье функции $f(\varphi, y, h)$ такой, что $\pi_{y} f(\varphi, y, h)=$ $\widehat{A}_{h}^{\prime}(\varphi, k, h)$, где оператор $\pi_{y}$ действует по переменной $y$.

Далее для краткости будем использовать запись вида $\widehat{A}_{h}(\varphi, h) \in S_{h \alpha}^{\infty}$.

При $x \in[-\pi / 2, \pi / 2], y \in[-\pi / 2, \pi / 2]$ имеет место неравенство

$$
|\sin x| \leqslant|\sin (y-x)|+|\sin y| .
$$

Из (1.11) следует оценка

$$
\frac{1}{h_{i}}\left|\sin \frac{\varphi_{i} h_{i}}{2}\right| \leqslant \frac{1}{h_{i}}\left|\sin \frac{h_{i}\left(\psi_{i}-\varphi_{i}\right)}{2}\right|+\frac{1}{h_{i}}\left|\sin \frac{\psi_{i} h_{i}}{2}\right|
$$

при $\varphi_{i}, \psi_{i} \in\left[-\pi / h_{i}, \pi / h_{i}\right], i=1, \ldots, n$.

Суммируя неравенства (1.12), затем прибавляя единицу, получаем

$$
1+\sum_{i=1}^{n} \frac{1}{h_{i}}\left|\sin \frac{\varphi_{i} h_{i}}{2}\right| \leqslant\left(1+\sum_{i=1}^{n} \frac{1}{h_{i}}\left|\sin \frac{\psi_{i} h_{i}}{2}\right|\right)\left(1+\sum_{i=1}^{n} \frac{1}{h_{i}}\left|\sin \frac{h_{i}\left(\psi_{i}-\varphi_{i}\right)}{2}\right|\right) .
$$

Из последнего неравенства следует оценка

$$
\left(1+\sum_{i=1}^{n} \frac{1}{h_{i}}\left|\sin \frac{h_{i}\left(\psi_{i}-\varphi_{i}\right)}{2}\right|\right)^{-N} \leqslant\left[\frac{1+\sum_{i=1}^{n} \frac{1}{h_{i}}\left|\sin \frac{\psi_{i} h_{i}}{2}\right|}{1+\sum_{i=1}^{n} \frac{1}{h_{i}}\left|\sin \frac{\varphi_{i} h_{i}}{2}\right|}\right]^{N}
$$

Ввиду симметрии $\varphi$ и $\psi$ из (1.13) получим

$$
\left(1+\sum_{i=1}^{n} \frac{1}{h_{i}}\left|\sin \frac{h_{i}\left(\psi_{i}-\varphi_{i}\right)}{2}\right|\right)^{-N} \leqslant\left[\frac{1+\sum_{i=1}^{n} \frac{1}{h_{i}}\left|\sin \frac{\varphi_{i} h_{i}}{2}\right|}{1+\sum_{i=1}^{n} \frac{1}{h_{i}}\left|\sin \frac{\psi_{i} h_{i}}{2}\right|}\right]^{N}
$$

ПреДЛОЖенИЕ 1.2. Семейство n.p.o. $A_{h} \in S_{h \alpha}$ с переменными ступенчатыми символами $\widehat{A}_{h}(\varphi, k, h)$ равномерно ограниченно отображает пространство $M(r, h)$ в $M(r-\alpha, h)$ для любого $r$ при $|h| \leqslant h_{0}$. 
ДокАЗАтЕльство. Рассмотрим ступенчатое преобразование Фурье функции $V(x, h)$ такой, что

$$
V(k)=\pi V(x, h)=\frac{1}{(2 \pi)^{n}} \int_{D_{h}} \widehat{A}_{h}(\varphi, k, h) \widehat{u}_{h}(\varphi) e^{-i(k \varphi, h)} d \varphi
$$

где $\widehat{u}_{h}(\varphi)$ - ступенчатое преобразование Фурье функции $u(x, h) \in M(r, h)$.

Из определения 1.5 следует

$$
\begin{aligned}
\widehat{V}_{h}(\psi) & =\Omega_{h} \sum_{k \in \mathbb{Z}_{n}} V(k) e^{i(k \psi, h)} \\
& =\frac{1}{(2 \pi)^{n}} \int_{D_{h}}\left(\Omega_{h} \sum_{k \in \mathbb{Z}_{n}} \widehat{A}_{h}(\varphi, k, h) e^{i(k(\psi-\varphi), h)} \widehat{u}_{h}(\varphi)\right) d \varphi \\
& =\frac{1}{(2 \pi)^{n}} \int_{D_{h}} \widehat{A}_{h}^{\prime}(\varphi, \psi-\varphi, h) \widehat{u}_{h}(\varphi) d \varphi
\end{aligned}
$$

где $\widehat{A}_{h}^{\prime}(\varphi, \psi, h)=\Omega_{h} \sum_{k \in \mathbb{Z}_{n}} \widehat{A}_{h}(\varphi, k, h) e^{i(k \psi, h)}$.

Из (1.11) следует оценка

$$
\left|\widehat{V}_{h}(\psi)\right| \leqslant C_{N} \int_{D_{h}} \frac{\left(1+\sum_{i=1}^{n} \frac{1}{h_{i}}\left|\sin \frac{\varphi_{i} h_{i}}{2}\right|\right)^{\alpha}\left|\widehat{u}_{h}(\varphi)\right| d \varphi}{\left(1+\sum_{i=1}^{n} \frac{1}{h_{i}}\left|\sin \frac{\left(\psi_{i}-\varphi_{i}\right) h_{i}}{2}\right|\right)^{N}}
$$

Из неравенства (1.15) получим

$$
\begin{aligned}
(1 & \left.+\sum_{i=1}^{n} \frac{1}{h_{i}}\left|\sin \frac{\psi_{i} h_{i}}{2}\right|\right)^{r-\alpha}\left|\widehat{V}_{h}(\psi)\right| \\
& \leqslant C_{N} \int_{D_{h}} \frac{\left(1+\sum_{i=1}^{n} \frac{1}{h_{i}}\left|\sin \frac{\psi_{i} h_{i}}{2}\right|\right)^{r-\alpha}\left(1+\sum_{i=1}^{n} \frac{1}{h_{i}}\left|\sin \frac{\varphi_{i} h_{i}}{2}\right|\right)^{\alpha}}{\left(1+\sum_{i=1}^{n} \frac{1}{h_{i}}\left|\sin \frac{\left(\psi_{i}-\varphi_{i}\right) h_{i}}{2}\right|\right)^{N}} \cdot\left|\widehat{u}_{h}(\varphi)\right| d \varphi
\end{aligned}
$$

Из неравенств $(1.13),(1.14)$ и (1.16) вытекает оценка

$$
\begin{aligned}
(1 & \left.+\sum_{i=1}^{n} \frac{1}{h_{i}}\left|\sin \frac{\psi_{i} h_{i}}{2}\right|\right)^{r-\alpha}\left|\widehat{V}_{h}(\psi)\right| \\
& \leqslant C_{N} \int_{D_{h}} \frac{\left(1+\sum_{i=1}^{n} \frac{1}{h_{i}}\left|\sin \frac{\psi_{i} h_{i}}{2}\right|\right)^{r} \cdot\left|\widehat{u}_{h}(\varphi)\right| d \varphi}{\left(1+\sum_{i=1}^{n} \frac{1}{h_{i}}\left|\sin \frac{\left(\psi_{i}-\varphi_{i}\right) h_{i}}{2}\right|\right)^{N-|r-\alpha|}}
\end{aligned}
$$

При достаточно большом $N$ имеет место оценка

$$
\int_{D_{h}} \frac{d \varphi}{\left(1+\sum_{i=1}^{n} \frac{1}{h_{i}}\left|\sin \frac{\left(\psi_{i}-\varphi_{i}\right) h_{i}}{2}\right|\right)^{N-|r-\alpha|}} \leqslant C_{1}
$$

где $C_{1}$ не зависит от $h=\left(h_{1}, \ldots, h_{n}\right)$ и $\psi=\left(\psi_{1}, \ldots, \psi_{n}\right)$. 
Из (1.17) и (1.18) следует неравенство

$$
\begin{aligned}
\|V(x, h)\|_{M(r-\alpha, h)}^{2} \leqslant C_{2} \int_{D_{h}}\left(1+\sum_{i=1}^{n} \frac{1}{h_{i}}\left|\sin \frac{\psi_{i} h_{i}}{2}\right|\right)^{2(r-\alpha)}\left|\widehat{V}_{h}(\psi)\right|^{2} d \psi \\
\leqslant C_{3} \int_{D_{h}}\left[\int_{D_{h}} \frac{\left(1+\sum_{i=1}^{n} \frac{1}{h_{i}}\left|\sin \frac{\varphi_{i} h_{i}}{2}\right|\right)^{r}\left|\widehat{u}_{h}(\varphi)\right| d \varphi}{\left(1+\sum_{i=1}^{n} \frac{1}{h_{i}}\left|\sin \frac{\left(\psi_{i}-\varphi_{i}\right) h_{i}}{2}\right|\right)^{N-|r-\alpha|}}\right] d \psi \\
\leqslant C_{4} \int_{D_{h}}\left[\int_{D_{h}} \frac{\left(1+\sum_{i=1}^{n} \frac{1}{h_{i}}\left|\sin \frac{\varphi_{i} h_{i}}{2}\right|\right)^{2 r}\left|\widehat{u}_{h}(\varphi)\right|^{2} d \varphi}{\left(1+\sum_{i=1}^{n} \frac{1}{h_{i}}\left|\sin \frac{\left(\psi_{i}-\varphi_{i}\right) h_{i}}{2}\right|\right)^{N-|r-\alpha|}}\right] d \psi \\
\leqslant C_{5} \int_{D_{h}}\left(1+\sum_{i=1}^{n} \frac{1}{h_{i}}\left|\sin \frac{\varphi_{i} h_{i} \mid}{2}\right|\right)^{2 r}\left|\widehat{u}_{h}(\varphi)\right|^{2} \\
\quad \times\left[\int_{D_{h}} \frac{d \psi}{\left.\left(1+\sum_{i=1}^{n} \frac{1}{h_{i}}\left|\sin \frac{\left(\psi_{i}-\varphi_{i}\right) h_{i}}{2}\right|\right)^{N-|r-\alpha|}\right] d \varphi}\right. \\
\leqslant C_{6}\|u(x, h)\|_{M(r, h)}^{2} .
\end{aligned}
$$

Из последнего неравенства следует утверждение предложения 1.2.

СлЕДСТвИЕ 1.1. Пусть $u(x) \in C_{0}^{\infty}\left(\mathbb{R}_{n}\right), u(x, h)=\prod_{h}^{*} u(x)\left(\prod_{h}^{*}-\right.$ точечный проектор). Тогда семейство операторов $A_{h}$, определенных равенством

$$
A_{h} a(x, h)=u(x, h) \cdot a(x, h),
$$

отображает пространство $M(r, h)$ в $M(r, h)$ равномерно ограниченно при $|h| \leqslant h_{0}, h_{0}>0$ - некоторое число.

ДоказАТЕЛЬСтво. Оператор $A_{h}$ можно представить в виде п.р.о.

$$
\pi A_{h} a(x, h)=\frac{1}{(2 \pi)^{n}} \int_{D_{h}} u(k, h) \widehat{a}_{h}(\varphi) e^{-i(k \varphi, h)} d \varphi
$$

причем $u(k, h)$ является символом п.р.о. $A_{h}$. Согласно замечанию 1.1 п.р.о. $A_{h}$ принадлежит классу $S_{h 0}$. Отсюда и из предложения 1.2 следует утверждение следствия 1.1.

СлЕДСТВИЕ 1.2. Если и $(x, h)=\prod_{h} u(x)$, әде $\prod_{h}-$ интегральный проектор, то утверждение следствия 1.1 сохраняется.

Теорема 1.1. Пусть $A_{h}$ - семейство п.р.о. с постоянными ступенчатыми символами $\widehat{A}_{h}(\varphi, h) \in S_{h \alpha}^{\infty}$. Тогда существует такое $h_{0}>0$, что при $|h| \leqslant h_{0}$ будет выполняться оченка

$$
\left\|\prod_{h}^{*} \alpha(x) A_{h}\left[\left(\prod_{h}^{*} \beta(x)\right) V(x, h)\right]\right\|_{M(r, h)} \leqslant C_{r s}(\delta)\|V(x, h)\|_{M(s, h)},
$$

где $V(x, h) \in M(s, h), s$ - произвольно, $r \geqslant 0$ - произвольно, $\alpha(x), \beta(x) \in$ $C_{0}^{\infty}\left(\mathbb{R}_{n}\right), \delta>0$ - расстояние между носителями функций $\alpha(x), \beta(x), \prod_{h}^{*}$ - точечный проектор. 
ДОКАЗАТЕЛЬСТВО. ОбозНачим

$$
f(k)=\pi \prod_{h}^{*} \alpha(x) A_{h}\left[\left(\prod_{h}^{*} \beta(x)\right) V(x, h)\right],
$$

и пусть $V(k h, h)$ - значение ступенчатой функции $V(x, h)$ на ячейке $D(k, h)$.

Тогда справедливо представление

$$
\begin{aligned}
f(k)= & \frac{1}{(2 \pi)^{n}} \int_{D_{h}} \alpha(k h) \widehat{A}_{h}(\varphi, h) \\
& \times\left(\sum_{m} \beta(m h) V(m h, h) e^{i(m \varphi, h)} \cdot \Omega_{h}\right) e^{-i(k \varphi, h)} d \varphi \\
= & \frac{1}{(2 \pi)^{n}} \sum_{m} V(m h, h) \int_{D_{h}} \alpha(k h) \beta(m h) \widehat{A}_{h}(\varphi, h) e^{i((m-k) \varphi, h)} d \varphi .
\end{aligned}
$$

Имеет место тождество

$$
\widehat{\Delta}_{\varphi}^{N} e^{i((m-k) \varphi, h)}=|(m-k) h|^{2 N} e^{i((m-k) \varphi, h)},
$$

где $\widehat{\Delta}_{\varphi}^{N}=(-1)^{N} \Delta_{\varphi}^{N}, \Delta_{\varphi}^{N}$ - итерированный оператор Лапласа по переменной $\varphi$.

Используя тождество (1.22), получаем равенство

$$
\begin{aligned}
\int_{D_{h}} & \alpha(k h) \beta(m h) \widehat{A}_{h}(\varphi, h) e^{i((m-k) \varphi, h)} d \varphi \\
& =\int_{D_{h}} \frac{\alpha(k h) \beta(m h) \widehat{A}_{h}(\varphi, h)}{|(m-k) h|^{2 N}} \cdot\left(\widehat{\Delta}_{\varphi}^{N} e^{i((m-k) \varphi, h)}\right) d \varphi \\
& =\int_{D_{h}} \frac{\alpha(k h) \beta(m h)}{|(m-k) h|^{2 N}}\left[\widehat{\Delta}_{\varphi}^{N} \widehat{A}_{h}(\varphi, h)\right] \cdot e^{i((m-k) \varphi, h)} d \varphi=: E_{N}(k, m) .
\end{aligned}
$$

Пусть $V(x, h) \in M_{h}^{\infty}$. Тогда из (1.21) и (1.23) следует соотношение

$$
f(k)=\frac{1}{(2 \pi)^{n}} \Omega_{h} \sum_{m} V(m h, h) E_{N}(k, m) .
$$

Применяя равенство Парсеваля по переменной $m$ и выполняя ступенчатое преобразование Фурье по переменной $k$ в соотношении (1.24), получаем

$$
\widehat{f}_{h}\left(\sigma^{\prime}\right)=\frac{1}{(2 \pi)^{2 n}} \int_{D_{h}} \overline{\widehat{V}_{h}\left(\sigma^{\prime \prime}\right)} \widehat{E}_{N}\left(\sigma^{\prime}, \sigma^{\prime \prime}\right) d \sigma^{\prime \prime},
$$

где $\widehat{f}_{h}\left(\sigma^{\prime}\right), \widehat{V}_{h}\left(\sigma^{\prime \prime}\right), \widehat{E}_{N}\left(\sigma^{\prime}, \sigma^{\prime \prime}\right)$ - ступенчатые преобразования Фурье функций $f(x, h), V_{h}(x, h), E_{N}(x, y, h)$ таких, что $\pi f(x, h)=f(k), \pi V(x, h)=V(k h, h)$, $\pi E_{N}(x, y, h)=E_{N}(k, v) ; \pi-$ стандартный оператор (см. определение 1.2). Обозначим

$$
\begin{gathered}
C_{1}(m-k)=\int_{D_{h}}\left[\widehat{\Delta}_{\varphi}^{N} \widehat{A}_{h}(\varphi, h) e^{i((m-k) \varphi, h)}\right] d \varphi, \\
C_{2}(m, k)=\frac{\alpha(k h) \beta(m h)}{|(m-k) h|^{2 N}} .
\end{gathered}
$$


Заметим, что сушествует $h_{0}>0$ такое, что при $|h| \leqslant h_{0}$ функция $C_{2}(x, y, h)$, $\pi C_{2}(x, y, h)=C_{2}(m, k)$, принадлежит $M(r, h) \forall r>0$, причем выполняется неравенство

$$
\left\|C_{2}(x, y, h)\right\|_{M(r, h)} \leqslant C_{r}(\delta),
$$

где $C_{r}(\delta)$ не зависит от $h=\left(h_{1}, \ldots, h_{n}\right)$.

Исходя из обозначений (1.26) и определения ступенчатого преобразования $\Phi$ рье получаем

$$
\widehat{E}_{N}\left(\sigma^{\prime}, \sigma^{\prime \prime}\right)=\Omega_{h}^{2} \sum_{k} \sum_{m} C_{1}(m-k) C_{2}(m, k) e^{i\left[\left(k \sigma^{\prime}, h\right)+\left(m \sigma^{\prime \prime}, h\right)\right]} .
$$

Ввиду финитности функции $C_{2}(m, k)$ в правой части $(1.28)$ конечное число слагаемых.

Сделаем замену дискретных переменных

$$
\left\{\begin{array} { l } 
{ m _ { i } - k _ { i } = p _ { i } , } \\
{ k _ { i } = l _ { i } , } \\
{ i = 1 , \ldots , n , }
\end{array} \Rightarrow \left\{\begin{array}{l}
m_{i}=p_{i}+l_{i} \\
k_{i}=l_{i}, \\
i=1, \ldots, n
\end{array}\right.\right.
$$

Отсюда следует

$$
C_{1}(m-k)=C_{1}(p), \quad C_{2}(m, k)=\frac{\alpha(l h) \beta((p+l) h)}{|p h|^{2 N}}=C_{2}^{*}(l, p) .
$$

Кроме того, сделаем замену $\Theta_{i}^{\prime}=\sigma_{i}^{\prime}+\sigma_{i}^{\prime \prime}, \Theta_{i}^{\prime \prime}=\sigma_{i}^{\prime \prime}, i=1, \ldots, n$. Отсюда получим соотношение

$$
\left(k \sigma^{\prime}, h\right)+\left(m \sigma^{\prime \prime}, h\right)=\left(l \Theta^{\prime}, h\right)+\left(p \Theta^{\prime \prime}, h\right)
$$

Используя формулы (1.28)-(1.31), получаем

$$
\begin{aligned}
\widehat{E}_{N}\left(\Theta^{\prime}-\Theta^{\prime \prime}, \Theta^{\prime \prime}\right) & =\Omega_{h}^{2} \sum_{p} \sum_{l} C_{1}(p) C_{2}^{*}(l, p) e^{i\left[\left(l \Theta^{\prime}, h\right)+\left(p \Theta^{\prime \prime}, h\right)\right]} \\
& =\Omega_{h}^{2} \sum_{p} C_{1}(p)\left[\Omega_{h}^{2} \sum_{l} C_{2}^{*}(l, p) e^{i\left(l \Theta^{\prime}, h\right)}\right] e^{i\left(p \Theta^{\prime \prime}, h\right)} \\
& =\Omega_{h}^{2} \sum_{p} C_{1}(p)\left[\widehat{C}_{2 h}^{*}\left(\Theta^{\prime}, p\right)\right] e^{i\left(p \Theta^{\prime \prime}, h\right)} \\
& =\frac{1}{(2 \pi)^{n}} \int_{D_{h}} \widehat{C}_{2 h}^{*}\left(\Theta^{\prime}, \Theta^{\prime \prime}-\beta\right) \widehat{C}_{1 h}(\beta) d \beta
\end{aligned}
$$

Согласно условию теоремы и определению 1.10 для функции $\widehat{C}_{1 h}(\beta)$ справедливо неравенство

$$
\left|\widehat{C}_{1 h}(\beta)\right| \leqslant C_{N}\left(1+\sum_{i=1}^{n} \frac{1}{h_{i}}\left|\sin \frac{\beta_{i} h_{i}}{2}\right|\right)^{\alpha-2 N} .
$$


В силу предложения 1.1 для функции $C_{2 h}^{*}\left(\Theta^{\prime}, \Theta^{\prime \prime}\right)$ будет выполняться неравенство при $|h| \leqslant h_{0}$

$$
\begin{aligned}
\left|\widehat{C}_{2 h}^{*}\left(\Theta^{\prime}, \Theta^{\prime \prime}\right)\right| & \leqslant \frac{C_{k}(\delta)}{\left(1+\sum_{i=1}^{n} \frac{1}{h_{i}}\left|\sin \frac{\Theta_{i}^{\prime} h_{i}}{2}\right|+\sum_{i=1}^{n} \frac{1}{h_{i}}\left|\sin \frac{\Theta_{i}^{\prime \prime} h_{i}}{2}\right|\right)^{4 k}} \\
& \leqslant \frac{C_{k}(\delta)}{\left(1+\sum_{i=1}^{n} \frac{1}{h_{i}}\left|\sin \frac{\Theta_{i}^{\prime} h_{i}}{2}\right|\right)^{2 k} \cdot\left(1+\sum_{i=1}^{n} \frac{1}{h_{i}}\left|\sin \frac{\Theta_{i}^{\prime \prime} h_{i}}{2}\right|\right)^{2 k}},
\end{aligned}
$$

$-\pi / h_{i} \leqslant \Theta_{i}^{\prime}, \Theta_{i}^{\prime \prime} \leqslant \pi / h_{i}, i=1, \ldots, n ; k-$ любое.

Из неравенств (1.13), (1.14) и (1.34) следует оценка

$$
\left|\widehat{C}_{2 h}^{*}\left(\Theta^{\prime}, \Theta^{\prime \prime}-\beta\right)\right| \leqslant \frac{C_{k}(\delta)\left(1+\sum_{i=1}^{n} \frac{1}{h_{i}}\left|\sin \frac{\beta_{i} h_{i}}{2}\right|\right)^{2 k+\alpha-2 N}}{\left(1+\sum_{i=1}^{n} \frac{1}{h_{i}}\left|\sin \frac{\Theta_{i}^{\prime} h_{i}}{2}\right|\right)^{2 k} \cdot\left(1+\sum_{i=1}^{n} \frac{1}{h_{i}}\left|\sin \frac{\Theta_{i}^{\prime \prime} h_{i}}{2}\right|\right)^{2 k}}
$$

Выбрав $N$ так, чтобы $2 k+\alpha-2 N \leqslant 0$, имеем

$$
\widehat{E}_{N}\left(\Theta^{\prime}-\Theta^{\prime \prime}, \Theta^{\prime \prime}\right) \leqslant \frac{C_{1 k}(\delta)}{\left(1+\sum_{i=1}^{n} \frac{1}{h_{i}}\left|\sin \frac{\Theta_{i}^{\prime} h_{i}}{2}\right|\right)^{2 k} \cdot\left(1+\sum_{i=1}^{n} \frac{1}{h_{i}}\left|\sin \frac{\Theta_{i}^{\prime \prime} h_{i}}{2}\right|\right)^{2 k}} .
$$

Используя (1.36), легко получить оценку

$$
\left|\widehat{E}_{N}\left(\sigma^{\prime}, \sigma^{\prime \prime}\right)\right| \leqslant \frac{C_{2 k}(\delta)}{\left(1+\sum_{i=1}^{n} \frac{1}{h_{i}}\left|\sin \frac{\sigma_{i}^{\prime \prime} h_{i}}{2}\right|\right)^{k / 2} \cdot\left(1+\sum_{i=1}^{n} \frac{1}{h_{i}}\left|\sin \frac{\sigma_{i}^{\prime} h_{i}}{2}\right|\right)^{k / 2}} .
$$

Из оценок (1.25) и (1.37) следует неравенство

$$
\begin{aligned}
\|f(x, h)\|_{M(r, h)}^{2} & =\int_{D_{h}}\left(1+\left|\sigma^{\prime}\right|\right)^{2 r}\left|\widehat{f}_{h}\left(\sigma^{\prime}\right)\right|^{2} d \sigma^{\prime} \\
& \leqslant C \int_{D_{h}}\left(1+\left|\sigma^{\prime}\right|\right)^{2 r} \cdot\left|\int_{D_{h}}\right| \widehat{V}_{h}\left(\sigma^{\prime \prime}\right)|\cdot| \widehat{E}_{N}\left(\sigma^{\prime}, \sigma^{\prime \prime}\right)\left|d \sigma^{\prime \prime}\right| d \sigma^{\prime} \\
& \leqslant C \int_{D_{h}}\left(1+\left|\sigma^{\prime}\right|\right)^{2 r}\left(\int_{D_{h}}\left|\widehat{V}_{h}\left(\sigma^{\prime \prime}\right)\right|^{2} \cdot\left|\widehat{E}_{N}\left(\sigma^{\prime}, \sigma^{\prime \prime}\right)\right| d \sigma^{\prime \prime}\right) d \sigma^{\prime} \\
& \leqslant C_{3 k}(\delta) \int_{D_{h}} \frac{\left(1+\sigma^{\prime}\right)^{2 r}}{\left(1+\left|\sigma^{\prime}\right|\right)^{k / 2}}\left(\int_{D_{h}} \frac{\left|\widehat{V}_{h}\left(\sigma^{\prime \prime}\right)\right|^{2}}{\left(1+\left|\sigma^{\prime \prime}\right|\right)^{k / 2}} d \sigma^{\prime \prime}\right) d \sigma^{\prime}
\end{aligned}
$$

Выбрав $k$ достаточно большим (в зависимости от $r$ и $s$ ), получим оценку

$$
\|f(x, h)\|_{M(r, h)}=C_{r s}(\delta)\|V(x, h)\|_{M(s, h)},
$$

где константа $C_{r s}(\delta)$ не зависит от $h$. Итак, теорема 1.1 доказана.

Свойство, доказанное в теореме 1.1, будем называть свойством псевдолокальности п.р.о. класса $S_{h \alpha}^{\infty}$ с постоянньми ступенчатьми символами. 
Рассмотрим семейство п.р.о. $A_{h}$ с постоянными ступенчатьми символами $\widehat{A}_{h}(\varphi, h)$, принадлежащее классу $S_{h \alpha}^{\infty}$. Пусть $\alpha(x) \in C_{0}^{\infty}\left(\mathbb{R}_{n}\right)$, определим семейство операторов $B_{h}$ соотношением

$$
B_{h} u(x, h)=\alpha(x, h) u(x, h)
$$

где $u(x, h) \in M_{h}^{\infty}, \alpha(x, h)=\prod_{h}^{*} \alpha(x)$.

Семейство операторов $B_{h}$ можно представить в виде п.р.о.

$$
\pi B_{h} u(x, h)=\frac{1}{(2 \pi)^{n}} \int_{D_{h}} \pi \alpha(x, h) \widehat{u}_{h}(\varphi) e^{-i(k \varphi, h)} d \varphi
$$

где $\widehat{A}(\varphi, k, h)=\pi \alpha(x, h)$ - ступенчатый символ п.р.о.; $\widehat{u}_{h}(\varphi)$ - ступенчатое преобразование Фурье функции $u(x, h)$.

ПРЕДЛОЖЕНИЕ 1.3. Onератор $T_{h}=A_{h} B_{h}-B_{h} A_{h}$ представи́м в виде п.p.o. класса $S_{h(\alpha-1)}$.

ДоказАтЕЛьство. Оператор $A_{h} B_{h} u(x, h)$ запишем в виде п.р.о.

$$
\pi B_{h} A_{h} u(x, h)=\frac{1}{(2 \pi)^{n}} \int_{D_{h}} \pi \alpha(x, h) \widehat{A}_{h}(\varphi, h) \widehat{u}_{h}(\varphi) e^{-i(k \varphi, h)} d \varphi
$$

Рассмотрим ступенчатое преобразование Фурье функции $B_{h} u(x, h)=\alpha(x, h) \times$ $u(x, h)=V(x, h)$ :

$$
\begin{aligned}
\widehat{V}_{h}(\varphi) & =\Omega_{h} \sum_{k \in \mathbb{Z}_{n}} \alpha(k) u(k) e^{i(k \varphi, h)} \\
& =\Omega_{h} \sum_{k \in \mathbb{Z}_{n}} \alpha(k) \frac{1}{(2 \pi)^{n}} \int_{D_{h}} \widehat{u}_{h}(\eta) e^{-i(k \eta, h)} d \eta \cdot e^{i(k \varphi, h)} \\
& =\frac{1}{(2 \pi)^{n}} \int_{D_{h}} \widehat{u}_{h}(\eta) \widehat{\alpha}(\varphi-\eta) d \eta
\end{aligned}
$$

Отсюда следует, что оператор $A_{h} B_{h}$ можно представить в виде п.р.о.

$$
\begin{aligned}
\pi A_{h} B_{h} u(x, h)=\frac{1}{(2 \pi)^{2 n}} \int_{D_{h}} \int_{D_{h}} \widehat{A}_{h}(\varphi, h) \widehat{u}_{h}(\eta) \widehat{\alpha}_{h}(\varphi-\eta) d \eta d \varphi \\
=\frac{1}{(2 \pi)^{n}} \int_{D_{h}} \widehat{u}_{h}(\eta) e^{-i(k \eta, h)} \\
\quad \times\left[\frac{1}{(2 \pi)^{n}} \int_{D_{h}} \widehat{A}_{h}(\sigma+\eta, h) \widehat{\alpha}_{h}(\sigma) e^{-i(k \sigma, h)} d \sigma\right] d \eta
\end{aligned}
$$

Из (1.39) вытекает соотношение

$$
\begin{aligned}
& \pi B_{h} A_{h} u(x, h) \\
& \quad=\frac{1}{(2 \pi)^{n}} \int_{D_{h}} \widehat{u}_{h}(\eta) e^{-i(k \eta, h)}\left[\frac{1}{(2 \pi)^{n}} \int_{D_{h}} \widehat{A}_{h}(\eta, h) \widehat{\alpha}_{h}(\sigma) e^{-i(k \sigma, h)} d \sigma\right] d \eta .
\end{aligned}
$$


Из (1.40) и (1.41) получаем представление для оператора $T_{h}$

$$
\begin{aligned}
& \pi T_{h} u(x, h)=\frac{1}{(2 \pi)^{n}} \int_{D_{h}} \widehat{u}_{h}(\eta) e^{-i(k \eta, h)} \\
& \quad \times\left[\frac{1}{(2 \pi)^{n}} \int_{D_{h}}\left[\widehat{A}_{h}(\sigma+\eta, h)-\widehat{A}_{h}(\eta, h)\right] \widehat{\alpha}_{h}(\sigma) e^{-i(k \sigma, h)} d \sigma\right] d \eta .
\end{aligned}
$$

Из (1.42) следует, что символ $\widehat{T}_{h}(\eta, k, h)$ оператора $T_{h}$ задается соотношением

$$
\widehat{T}_{h}(\eta, k, h)=\frac{1}{(2 \pi)^{n}} \int_{D_{h}}\left[\widehat{A}_{h}(\sigma+\eta, h)-\widehat{A}_{h}(\eta, h)\right] \widehat{\alpha}_{h}(\sigma) e^{-i(k \sigma, h)} d \sigma .
$$

Докажем, что функция $R_{h}(\eta, \sigma)=\widehat{A}_{h}(\sigma+\eta, h)-\widehat{A}_{h}(\eta, h)$ удовлетворяет неравенству

$$
\left|R_{h}(\eta, \sigma)\right| \leqslant C\left(1+\sum_{i=1}^{n} \frac{1}{h_{i}}\left|\sin \frac{\eta_{i} h_{i}}{2}\right|\right)^{\alpha-1} \cdot\left(1+\sum_{i=1}^{n} \frac{1}{h_{i}}\left|\sin \frac{\sigma_{i} h_{i}}{2}\right|\right)^{|\alpha|+1}
$$

где $C$ не зависит от $h ; \eta, \sigma \in D_{h}$.

Рассмотрим случай $|\sigma| \geqslant \frac{1}{2}(1+|\eta|), \sigma, \eta \in D_{h}$. Имеем

$$
\begin{aligned}
& \left|\widehat{A}_{h}(\eta, h)\right| \leqslant C\left(1+\sum_{i=1}^{n} \frac{1}{h_{i}}\left|\sin \frac{\eta_{i} h_{i}}{2}\right|\right)^{\alpha} \\
& \quad \leqslant C \frac{\left(1+\sum_{i=1}^{n} \frac{1}{h_{i}}\left|\sin \frac{\eta_{i} h_{i}}{2}\right|\right)^{\alpha}}{\left(1+\sum_{i=1}^{n} \frac{1}{h_{i}}\left|\sin \frac{\sigma_{i} h_{i}}{2}\right|\right)} \cdot\left(1+\sum_{i=1}^{n} \frac{1}{h_{i}}\left|\sin \frac{\sigma_{i} h_{i}}{2}\right|\right) \\
& \quad \leqslant C_{1}\left(1+\sum_{i=1}^{n} \frac{1}{h_{i}}\left|\sin \frac{\eta_{i} h_{i}}{2}\right|\right)^{\alpha-1}\left(1+\sum_{i=1}^{n} \frac{1}{h_{i}}\left|\sin \frac{\sigma_{i} h_{i}}{2}\right|\right)^{\alpha}
\end{aligned}
$$

Перейдем к оценке $A_{h}(\eta+\sigma, h)$. Так как $\eta, \sigma \in D_{h}$, то имеют место оценки

$$
-\frac{2 \pi}{h_{i}} \leqslant \eta_{i}+\sigma_{i} \leqslant \frac{2 \pi}{h_{i}}, \quad i=1, \ldots, n
$$

Из неравенств (1.46) следует, что множество значений индекса $i, 1 \leqslant i \leqslant n$, можно представить в виде объединения непересекающихся множеств $O_{j}(\eta, \sigma), j=1,2,3$, обладающих свойствами:

1) если $i \in O_{1}(\eta, \sigma)$, то

$$
-\frac{2 \pi}{h_{i}} \leqslant \eta_{i}+\sigma_{i} \leqslant-\frac{\pi}{h_{i}}
$$

2) если $i \in O_{2}(\eta, \sigma)$, то

$$
-\frac{\pi}{h_{i}} \leqslant \eta_{i}+\sigma_{i} \leqslant \frac{\pi}{h_{i}}
$$


3) если $i \in O_{3}(\eta, \sigma)$, то

$$
\frac{\pi}{h_{i}} \leqslant \eta_{i}+\sigma_{i} \leqslant \frac{2 \pi}{h_{i}}
$$

Обозначим $\Theta_{i}^{j}=\eta_{i}+\sigma_{i}$, если $i \in O_{j}(\eta, \sigma), j=1,2,3$. Тогда $\Theta^{j}=\left(\ldots, \Theta_{k}^{j}, \ldots\right)$ есть вектор, для которого все $k \in O_{j}(\eta, \sigma)$. Компонентами вектора $h^{j}$ являются величины $h_{i}$ такие, что $i \in O_{j}(\eta, \sigma)$. Для функции $\widehat{A}_{h}(\eta+\sigma, h)$ введем обозначение $\widehat{A}_{h}(\eta+\sigma, h)=\widehat{A}_{1 h}\left(\Theta^{1}, \Theta^{2}, \Theta^{3}\right)$. Очевидно, что имеет место соотношение

$$
\widehat{A}_{h}(\eta+\sigma, h)=\widehat{A}_{1 h}\left(\Theta^{1}+\frac{2 \pi}{h^{1}}, \Theta^{2}, \Theta^{3}-\frac{2 \pi}{h^{3}}\right) .
$$

Так как функция $\widehat{A}_{h}(\eta, h) \in S_{h \alpha}^{\infty}$, а переменные $\Theta^{1}+2 \pi / h^{1}, \Theta^{2}$ и $\Theta^{3}-2 \pi / h^{3}$ принадлежат $D_{h}$, то имеет место неравенство

$$
\begin{aligned}
\left|\widehat{A}_{h}(\eta+\sigma, h)\right| \leqslant\left(1+\sum_{i \in O_{1}} \frac{1}{h_{i}}\left|\sin \frac{\left(\eta_{i}+\sigma_{i}+2 \pi / h_{i}\right) h_{i}}{2}\right|\right. \\
\left.+\sum_{i \in O_{2}} \frac{1}{h_{i}}\left|\sin \frac{\left(\eta_{i}+\sigma_{i}\right) h_{i} \mid}{2}\right|+\sum_{i \in O_{3}} \frac{1}{h_{i}}\left|\sin \frac{\left(\eta_{i}+\sigma_{i}-2 \pi / h_{i}\right) h_{i}}{2}\right|\right)^{\alpha} \\
\leqslant C_{1}\left(1+\sum_{i=1}^{n}\left|\eta_{i}+\sigma_{i}\right|\right)^{\alpha} \leqslant C_{2}\left(1+\sum_{i=1}^{n}\left|\eta_{i}\right|\right)^{\alpha} \cdot\left(1+\sum_{i=1}^{n}\left|\sigma_{i}\right|\right)^{|\alpha|} .
\end{aligned}
$$

Из (1.50), используя неравенство $|\sigma| \geqslant \frac{1}{2}(1+|\eta|)$, получаем оценку

$$
\begin{aligned}
& \left|\widehat{A}_{h}(\eta+\sigma, h)\right| \leqslant C_{3}\left(1+\sum_{i=1}^{n}\left|\eta_{i}\right|\right)^{\alpha-1} \cdot\left(1+\sum_{i=1}^{n}\left|\sigma_{i}\right|\right)^{|\alpha|+1} \\
& \leqslant C_{4}\left(1+\sum_{i=1}^{n} \frac{1}{h_{i}}\left|\sin \frac{\eta_{i} h_{i}}{2}\right|\right)^{\alpha-1} \cdot\left(1+\sum_{i=1}^{n} \frac{1}{h_{i}}\left|\sin \frac{\sigma_{i} h_{i}}{2}\right|\right)^{|\alpha|+1} .
\end{aligned}
$$

Итак, в рассматриваемом случае из (1.45) и (1.51) следует оценка (1.44).

Рассмотрим теперь случай $|\sigma| \geqslant \frac{1}{2}(1+|\eta|), \sigma, \eta \in D_{h}$. Так как $\widehat{A}_{h}(\eta, h) \in S_{h \alpha}^{\infty}$, то, применяя теорему Лагранжа, аналогично (1.50) получаем

$$
\begin{aligned}
\left|\widehat{R}_{h}(\eta, \sigma)\right| & =\left|\widehat{A}_{h}(\eta+\sigma, h)-\widehat{A}_{h}(\eta, h)\right|=\left|\sum_{i=1}^{n} \frac{\partial \widehat{A}_{h}(\eta+t \sigma, h)}{\partial \eta_{i}} \sigma_{i}\right| \\
& \leqslant C_{1} \sum_{i=1}^{n}\left(1+\sum_{k=1}^{n} \frac{1}{h_{k}}\left|\sin \frac{\left(\eta_{k}+t \sigma_{k}\right) h_{k}}{2}\right|\right)^{\alpha-1}\left|\sigma_{i}\right| \\
& \leqslant C_{2}\left(1+\sum_{k=1}^{n} \frac{1}{h_{k}}\left|\sin \frac{\left(\eta_{k}+t \sigma_{k}\right) h_{k}}{2}\right|\right)^{\alpha-1}|\sigma|,
\end{aligned}
$$

где $0<t<1$. 
Из (1.52), используя условие $|\sigma| \geqslant \frac{1}{2}(1+|\eta|)$, получаем

$$
\begin{aligned}
\left|\widehat{R}_{h}(\eta, \sigma)\right| & \leqslant C_{3}(1+|\eta|)^{\alpha-1}|\sigma| \\
& \leqslant C_{3}\left(1+\sum_{i=1}^{n} \frac{1}{h_{i}}\left|\sin \frac{\eta_{i} h_{i}}{2}\right|\right)^{\alpha-1} \cdot\left(1+\sum_{i=1}^{n} \frac{1}{h_{i}}\left|\sin \frac{\sigma_{i} h_{i}}{2}\right|\right) .
\end{aligned}
$$

Итак, неравенство (1.44) доказано.

Из формулы (1.43) следует соотношение

$$
F_{h}\left(\widehat{T}_{h}(\eta, k, h)\right)=T_{h}^{\prime}(\eta, \sigma, h)=\left[\widehat{A}_{h}(\eta+\sigma, h)-\widehat{A}_{h}(\eta, h)\right] \widehat{\alpha}_{h}(\sigma) .
$$

Из предложения 1.1 и неравенства (1.44) вытекает оценка

$$
\left|T_{h}^{\prime}(\eta, \sigma, h)\right| \leqslant \frac{C_{N}\left(1+\sum_{i=1}^{n} \frac{1}{h_{i}}\left|\sin \frac{\eta_{i} h_{i}}{2}\right|\right)^{\alpha-1}}{\left(1+\sum_{i=1}^{n} \frac{1}{h_{i}}\left|\sin \frac{\sigma_{i} h_{i}}{2}\right|\right)^{N}}
$$

Из последнего неравенства и определения 1.11 следует предложение 1.3.

\section{§ 2. Псевдоразностные уравнения}

ОПреДЕЛЕНИЕ 2.1. Семейство псевдоразностных операторов $A_{h} \in S_{h \alpha}^{\infty}$ с постоянными ступенчатьми символами $\widehat{A}_{h}(\varphi, h)$ будем назьвать сильно эллиптическим, если вьполняется неравенство

$$
\left|\widehat{A}_{h}(\varphi, h)\right| \geqslant C\left(1+\sum_{i=1}^{n} \frac{1}{h_{i}}\left|\sin \frac{\varphi_{i} h_{i}}{2}\right|\right)^{\alpha}
$$

где $C$ не зависит от $h=\left(h_{1}, \ldots, h_{n}\right)$.

При помощи математической индукции доказывается следующее утверждение.

ПРЕДЛОЖЕНИЕ 2.1. Пусть $A_{h} \in S_{h \alpha}^{\infty}$ - сильно әллиптическое семейство п.р.о. Тогда семейство п.р.о. $T_{h}$, определенное соотношением

$$
T_{h} u(x, h)=\frac{1}{(2 \pi)^{n}} \int_{D_{h}} \frac{1}{\widehat{A}_{h}(\varphi, h)} \widehat{u}_{h}(\varphi) e^{i(k \varphi, h)} d \varphi
$$

является сильно әллиптическим семейством п.р.о. с символами $\widehat{T}_{h}(\varphi, h) \in$ $S_{h(-\alpha)}^{\infty}$

Рассмотрим ограниченный оператор $T$ конечного порядка, отображающий $\stackrel{\circ}{H}_{r}(\Omega)$ в $H_{-r}\left(\mathbb{R}_{n}\right)$, где $\Omega$ - нормальная допускающая разбиение область (см. [2]). Пусть устойчивое семейство операторов $T_{h}: \stackrel{\circ}{M}\left(r, h, \Omega_{1 h}\right) \rightarrow M(-r, h), r>0$, $\prod_{h}^{*}$-аппроксимирует в области $\Omega$ оператор $T$ (см. [2]). Кроме того, пусть семейство операторов $T_{h}$ является п.р.о. с постоянными ступенчатьми символами сильно эллиптического типа, причем их символы $\widehat{A}_{h}(\varphi, h) \in S_{h(2 r)}^{\infty}$. 
Рассмотрим уравнение

$$
p T u=f
$$

где $u(x) \in \stackrel{\circ}{H}_{r}(\Omega), f(x) \in H_{\lambda}(\Omega), \lambda>0, p$ - оператор сужения на область $\Omega$, т.е. уравнение (2.1) понимается следуюшим образом: $\forall V(x) \in \stackrel{\circ}{H}_{r}(\Omega)$ вьполняется равенство

$$
(T u, V)=(l f, V),
$$

где $l f \in H_{\lambda}\left(\mathbb{R}_{n}\right)$ - некоторое продолжение $f$ на $\mathbb{R}_{n}$.

ТЕОРЕМА 2.1. Пусть для уравнения (2.1) выполняются указанные условия. Приближенное решение уравнения (2.1) будем искать из системы

$$
p_{h} T_{h} a(x, h)=p_{h} \prod_{h} f(x)
$$

где $\prod_{h}$ - интегральный проектор, $p_{h}$ - оператор сужения на область $\Omega_{1 h}$. Тогда $\left(\prod_{h}^{*} \psi(x)\right) a(x, h) \in \stackrel{\circ}{M}\left(2 r+\lambda, h, \Omega_{1 h}\right) \quad \forall \psi(x) \in C_{0}^{\infty}\left(\mathbb{R}_{n}\right), \operatorname{supp} \psi(x) \subset \Omega$, $\prod_{h}^{*}-$ точечный проектор.

ДокАЗАтЕльство. Пусть $a(x, h)$ есть решение системы (2.3). Обозначим $f(x, h)=T_{h} a(x, h), f(x, h) \in M(-r, h)$. Согласно предложению 2.1 оператор $T_{h}^{-1}$ сильно эллиптический с постоянньм ступенчатым символом $1 / \widehat{A}_{h}(\varphi, h) \in$ $S_{h(-2 r)}^{\infty}$.

Рассмотрим произвольную функцию $\psi(x) \in C_{0}^{\infty}\left(\mathbb{R}_{n}\right)$, причем supp $\psi(x) \subset \Omega$. Возьмем $\alpha_{1}(x) \in C_{0}^{\infty}\left(\mathbb{R}_{n}\right)$ такую, что $\operatorname{supp} \alpha_{1}(x) \subset \Omega, \alpha_{1}(x)=1$ при $x \in$ $\operatorname{supp} \psi(x)$. Если обозначить $D_{1}=\left\{x: \alpha_{1}(x)=1\right\}$, то сушествует открытая область $\mathscr{Y}_{1} \subset D_{1}$ такая, что $\operatorname{supp} \psi(x) \subset \mathscr{Y}_{1}$. Отсюда следует, что сушествует $h_{0}>0$ такое, что при $|h|<h_{0} \operatorname{supp} \psi(x) \subset \Omega_{1 h}, \operatorname{supp} \alpha_{1}(x) \subset \Omega_{1 h}$. Далее рассмотрим

$$
\begin{aligned}
T_{h}^{-1}[ & \left.\left(\prod_{h}^{*} \alpha_{1}(x)\right) T_{h} a(x, h)\right]=T_{h}^{-1} T_{h}\left[\left(\prod_{h}^{*} \alpha_{1}(x)\right) a(x, h)\right] \\
& +T_{h}^{-1}\left[\left(\prod_{h}^{*} \alpha_{1}(x)\right) T_{h} a(x, h)-T_{h}\left(\prod_{h}^{*} \alpha_{1}(x)\right) a(x, h)\right] \\
= & T_{h}^{-1}\left[\left(\prod_{h}^{*} \alpha_{1}(x)\right) \prod_{h} f(x)\right] .
\end{aligned}
$$

Соотношение (2.4) можно переписать в виде

$$
\begin{aligned}
a(x, h) \prod_{h}^{*} \alpha_{1}(x)= & -T_{h}^{-1}\left[\left(\prod_{h}^{*} \alpha_{1}(x)\right) T_{h} a(x, h)-T_{h}\left(\prod_{h}^{*} \alpha_{1}(x)\right) a(x, h)\right] \\
& +T_{h}^{-1}\left[\left(\prod_{h}^{*} \alpha_{1}(x)\right) \prod_{h} f(x)\right] .
\end{aligned}
$$

Из предложения 2.1 и теоремы об интегральном проекторе [1] следует оценка

$$
\left\|\left(\prod_{h}^{*} \alpha_{1}(x)\right) \prod_{h} f(x)\right\|_{\stackrel{\circ}{M\left(\lambda, h, \Omega_{1 h}\right)}} \leqslant C_{\lambda}\|f(x)\|_{H_{\lambda}(\Omega)} .
$$


Так как $T_{h}^{-1}$ - сильно эллиптический оператор и его символ $1 / \widehat{A}_{h}(\varphi, h) \in S_{h(-2 r)}^{\infty}$, то $g(x, h)=T_{h}^{-1}\left[\left(\prod_{h}^{*} \alpha_{1}(x)\right) \prod_{h} f(x)\right] \in M(\lambda+2 r, h)$, причем равномерно по $h$ вьполняется оценка

$$
\|g(x, h)\|_{M(\lambda+2 r, h)} \leqslant C_{\lambda, r}\|f(x)\|_{H_{\lambda}(\Omega)} .
$$

По предложению 1.3 функция

$$
g_{1}(x, h)=T_{h}^{-1}\left[\left(\prod_{h}^{*} \alpha_{1}(x)\right) T_{h} a(x, h)-T_{h}\left(\prod_{h}^{*} \alpha_{1}(x)\right) a(x, h)\right] \in M(r+1, h) .
$$

Отсюда и из (2.5) следует, что $a(x, h) \prod_{h}^{*} \alpha_{1}(x) \in \stackrel{\circ}{M}\left(s, h, \Omega_{1 h}\right)$, где $s=\min (r+1$, $\lambda+2 r)$, причем выполняется неравенство

$$
\left\|a(x, h) \prod_{h}^{*} \alpha_{1}(x)\right\|_{\stackrel{\circ}{\circ\left(s, h, \Omega_{1 h}\right)}} \leqslant C_{\lambda, r}\|f\|_{H_{\lambda}(\Omega)} .
$$

Возьмем функцию $\alpha_{2}(x) \in C_{0}^{\infty}\left(\mathbb{R}_{n}\right)$, обладающую следующими свойствами. Пусть $D_{2}=\left\{x: \alpha_{2}(x)=1\right\}$. Тогда существует открытая область $\mathscr{Y}_{2} \subset D_{2}, \mathscr{Y}_{2} \supset$ $\operatorname{supp} \psi(x)$, такая, что $\operatorname{supp} \alpha_{2}(x) \subset \mathscr{Y}_{1}, \alpha_{2}(x) \cdot \alpha_{1}(x)=\alpha_{2}(x)$, и сушествует такое $h_{1}>0$, что при $|h|<h_{1}$ будет вьполняться $\left(\prod_{h}^{*} \alpha_{2}(x)\right) \cdot\left(\prod_{h}^{*} \alpha_{1}(x)\right)=\prod_{h}^{*} \alpha_{2}(x)$.

Далее рассмотрим

$$
\begin{aligned}
T_{h}^{-1}[ & \left.\left(\prod_{h}^{*} \alpha_{2}(x)\right) T_{h} a(x, h)\right] \\
= & \left(\prod_{h}^{*} \alpha_{2}(x)\right) a(x, h) \\
& +T_{h}^{-1}\left[\left(\prod_{h}^{*} \alpha_{2}(x)\right) T_{h}\left(\prod_{h}^{*} \alpha_{1}(x)\right) a(x, h)\right. \\
& \left.-T_{h}\left(\left(\prod_{h}^{*} \alpha_{1}(x)\right)\left(\prod_{h}^{*} \alpha_{2}(x)\right)\right) a(x, h)\right] \\
& +T_{h}^{-1}\left(\left(\prod_{h}^{*} \alpha_{2}(x)\right) T_{h}\left(\prod_{h}^{*}\left(\Theta(x)-\alpha_{1}(x)\right) a(x, h)\right)\right) \\
= & T_{h}^{-1}\left(\left(\prod_{h}^{*} \alpha_{2}(x)\right)\left(\prod_{h} f(x)\right)\right),
\end{aligned}
$$

где $\Theta(x) \in C_{0}^{\infty}\left(\mathbb{R}_{n}\right), \Theta(x)=1$ при $x \in \Omega$.

Так как носители функций $\prod_{h}^{*} \alpha_{2}(x)$ и $\prod_{h}^{*}\left(\Theta(x)-\alpha_{1}(x)\right)$ не пересекаются для всех $h,|h|<h_{1}$, то по свойству псевдолокальности (теорема 1.1) для любого $s$ существует такое $h_{2}>0$, что при $|h|<h_{2}$ выполняется оценка

$$
\left\|T_{h}^{-1}\left(\left(\prod_{h}^{*} \alpha_{2}(x)\right) T_{h}\left(\prod_{h}^{*}\left(\Theta(x)-\alpha_{1}(x)\right) a(x, h)\right)\right)\right\|_{M(s, h)} \leqslant C_{s \lambda}\|f(x)\|_{H_{\lambda}(\Omega)} .
$$

Кроме того, имеет место неравенство

$$
\left\|T_{h}^{-1}\left(\left(\prod_{h}^{*} \alpha_{2}(x)\right)\left(\prod_{h} f(x)\right)\right)\right\|_{M(\lambda+2 r, h)} \leqslant C_{1}\|f(x)\|_{H_{\lambda}(\Omega)} .
$$


Используя неравенство (2.7) и предложение 1.3, получаем

$$
\begin{aligned}
& \| T_{h}^{-1}\left[\left(\prod_{h}^{*} \alpha_{2}(x)\right) T_{h}\left(\prod_{h}^{*} \alpha_{1}(x)\right) a(x, h)\right. \\
& \left.\quad-T_{h}\left(\left(\prod_{h}^{*} \alpha_{1}(x)\right)\left(\prod_{h}^{*} \alpha_{2}(x)\right)\right) a(x, h)\right]\left\|_{M(r+2, h)} \leqslant C_{2}\right\| f(x) \|_{H_{\lambda}(\Omega)} .
\end{aligned}
$$

Из последних замечаний и представления $(2.8)$ следует, что $a(x, h) \prod_{h}^{*} \alpha_{2}(x) \in$ $\stackrel{\circ}{M}\left(s_{1}, h, \Omega_{1 h}\right)$, где $s_{1}=\min (r+2, \lambda+2 r)$, причем выполняется оценка

$$
\left\|a(x, h) \prod_{h}^{*} \alpha_{2}(x)\right\|_{\stackrel{\circ}{\circ\left(s_{1}, h, \Omega_{1 h}\right)}} \leqslant C_{\lambda, r}\|f(x)\|_{H_{\lambda}(\Omega)} .
$$

Рассуждая далее аналогично, получаем утверждение теоремы 2.1.

СлЕДСТВИЕ 2.1. При выполнении условий теоремы 2.1 для точного решения уравнения (2.1) имеем $\psi(x) u(x) \in \stackrel{\circ}{M}_{2 r+\lambda}(\Omega), \operatorname{supp} \psi(x) \subset \Omega$.

Доказательство следует из теоремы 2.1 и результатов работ [1], [2].

СлеДСТВИе 2.2. Если $f(x) \in C^{\infty}\left(\mathscr{Y}_{1}\right), \overline{\mathscr{Y}}_{1} \subset \Omega$, то в любой области $\mathscr{Y}_{2}, \overline{\mathscr{Y}}_{2} \subset \mathscr{Y}_{1}$, сходятся равномерно разностные отношения любого порядка приближенного решения уравнения (2.3).

Доказательство следует из теоремы 2.1 и теоремы вложения для пространств дробных отношений $M(r, h)$ (см. [1], [2]).

Теорема 2.2. Пусть для семейства операторов $T_{h}$ и оператора $T$ выполняются условия теоремы 2.1 ; оператор $F$ ограниченно отображает $\stackrel{\circ}{H}_{s}(\Omega)$ в $H_{k}\left(\mathbb{R}_{n}\right)$ при $k-s+2 r \geqslant \varepsilon, \varepsilon>0, s$ произвольно; ограниченное семейство операторов $F_{h}: \stackrel{\circ}{M}\left(s, h, \Omega_{1 h}\right) \rightarrow M(k, h), k-s+2 r \geqslant \varepsilon, s$ произвольно, $\prod_{h}^{*}$-аnпроксимирует в нормальной допускающей разбиение области $\Omega$ оператор $F$ конечного порядка; операторы $F_{h}$ принадлежсат классу $S_{h(2 r-\varepsilon)}^{\infty}$. Пусть, кроме того, сужение $р A$ оператора $A=T+F$ на $H_{r}(\Omega)$ имеет ядро, состоящее из одного нулевого әлемента. Приближенное решение уравнения

$$
p A=f
$$

находим из системы

$$
p_{h} A_{h} a(x, h)=p_{h} \prod_{h} f(x),
$$

əде $f(x) \in L_{2}(\Omega), f(x) \in H_{\lambda}\left(\mathscr{Y}_{1}\right), \overline{\mathscr{Y}}_{1} \subset \Omega, \lambda>0$.

Тогда $\forall \psi(x) \in C_{0}^{\infty}\left(\mathscr{Y}_{2}\right), \overline{\mathscr{Y}}_{2} \subset \mathscr{Y}_{1}$, имеем

$$
\left(\prod_{h}^{*} \psi(x)\right) a(x, h) \in \stackrel{\circ}{M}\left(r+2 \lambda, h, \Omega_{1 h}\right)
$$

при $|h|<h_{0}, h_{0}>0$, причем выполняется оченка

$$
\left\|\left(\prod_{h}^{*} \psi(x)\right) a(x, h)\right\|_{\stackrel{\circ}{M\left(2 r+\lambda, \Omega_{1 h}\right)}} \leqslant C_{\lambda, r}\left(\|f(x)\|_{L_{2}(\Omega)}+\|f(x)\|_{H_{\lambda}\left(\mathscr{Y}_{1}\right)}\right) .
$$


ДокаЗАТЕЛЬСтво. Пусть $a(x, h)$ есть решение системы (2.11). Обозначим $f(x, h)=A_{h} a(x, h), f(x, h) \in M(-r, h) . \quad$ Пусть $\psi(x) \in C_{0}^{\infty}\left(\mathscr{Y}_{2}\right)$, возьмем $\alpha_{1}(x) \in C_{0}^{\infty}\left(\mathscr{Y}_{1}\right)$, причем $\operatorname{supp} \alpha_{1}(x) \subset \mathscr{Y}_{1}, \alpha_{1}(x)=1$ при $x \in \operatorname{supp} \psi(x)$. Кроме того, если обозначить $D_{1}=\left\{x: \alpha_{1}(x)=1\right\}$, то сушествует открытая область $B_{1} \subset D_{1}$ такая, что $\operatorname{supp} \psi(x) \subset B_{1}$. Отсюда следует существование $h_{0}>0$ такого, что при $|h|<h_{0} \operatorname{supp} \psi(x) \subset \mathscr{Y}_{1 h}, \operatorname{supp} \alpha_{1}(x) \subset \mathscr{Y}_{1 h}$.

Далее рассмотрим

$$
\begin{aligned}
T_{h}^{-1}[ & \left.\left(\prod_{h}^{*} \alpha_{1}(x)\right) A_{h} a(x, h)\right] \\
= & T_{h}^{-1} T_{h}\left[\left(\prod_{h}^{*} \alpha_{1}(x)\right) a(x, h)\right] \\
& +T_{h}^{-1}\left[\left(\prod_{h}^{*} \alpha_{1}(x)\right) T_{h} a(x, h)-T_{h}\left(\prod_{h}^{*} \alpha_{1}(x)\right) a(x, h)\right] \\
& +T_{h}^{-1}\left[\left(\prod_{h}^{*} \alpha_{1}(x)\right) F_{h} a(x, h)\right] \\
= & T_{h}^{-1}\left[\left(\prod_{h}^{*} \alpha_{1}(x)\right) \prod_{h} f(x)\right] .
\end{aligned}
$$

Соотношение (2.13) можно переписать в виде

$$
\begin{aligned}
& \left(\prod_{h}^{*} \alpha_{1}(x)\right) a(x, h) \\
& \quad=T_{h}^{-1}\left[\left(\prod_{h}^{*} \alpha_{1}(x)\right) \prod_{h} f(x)\right]-T_{h}^{-1}\left[\left(\prod_{h}^{*} \alpha_{1}(x)\right) F_{h} a(x, h)\right] \\
& \quad-T_{h}^{-1}\left[\left(\prod_{h}^{*} \alpha_{1}(x)\right) T_{h} a(x, h)-T_{h}\left(\left(\prod_{h}^{*} \alpha_{1}(x)\right) a(x, h)\right)\right] .
\end{aligned}
$$

Из предложения 2.1 и теоремы об интегральном проекторе (см. [1]) вытекает неравенство

$$
\left\|\left(\prod_{h}^{*} \alpha_{1}(x)\right) \prod_{h} f(x)\right\|_{\stackrel{\circ}{\circ}\left(\lambda, h, \Omega_{1 h}\right)} \leqslant C_{\lambda}\|f(x)\|_{H_{\lambda}(\Omega)} .
$$

Так как $T_{h}^{-1}-$ сильно эллиптический оператор и его символ $1 / \widehat{T}_{h}(\varphi, h) \in$ $S_{h(-2 r)}^{\infty}$, то $g(x, h)=T_{h}^{-1}\left[\left(\prod_{h}^{*} \alpha_{1}(x)\right) \prod_{h} f(x)\right] \in M(\lambda+2 r, h)$, причем равномерно выполняется оценка

$$
\|g(x, h)\|_{M(\lambda+2 r, h)} \leqslant C_{\lambda, r}\|f(x)\|_{H_{\lambda}(\Omega)} .
$$

Функция $b(x, h)=\left(\prod_{h}^{*} \alpha_{1}(x)\right) F_{h} a(x, h) \in M(-r+\varepsilon, h)$. Отсюда следует, что функция $b_{1}(x, h)=T_{h}^{-1} b(x, h) \in M(r+\varepsilon, h)$, причем выполняется неравенство

$$
\left\|b_{1}(x, h)\right\|_{M(r+\varepsilon, h)} \leqslant C_{1}\|f(x)\|_{L_{1}(\Omega)},
$$

где $C_{1}$ не зависит от $h=\left(h_{1}, \ldots, h_{n}\right)$. 
По теореме о коммутаторе функция

$$
g_{1}(x, h)=T_{h}^{-1}\left[\left(\prod_{h}^{*} \alpha_{1}(x)\right) T_{h} a(x, h)-T_{h}\left(\left(\prod_{h}^{*} \alpha_{1}(x)\right) a(x, h)\right)\right] \in M(r+\varepsilon, h) .
$$

Из (2.13)-(2.16) следует, что $a(x, h) \prod_{h}^{*} \alpha_{1}(x) \in \stackrel{\circ}{M}\left(s_{1}, h, \Omega_{1 h}\right), s_{1}=\min (r+\varepsilon$, $\lambda+2 r)$, причем выполняется неравенство

$$
\left\|a(x, h) \prod_{h}^{*} \alpha_{1}(x)\right\|_{\stackrel{\circ}{M\left(s_{1}, h, \Omega_{1 h}\right)}} \leqslant C_{\lambda, r}^{(1)}\left(\|f(x)\|_{L_{2}(\Omega)}+\|f(x)\|_{H_{\lambda}\left(\mathscr{Y}_{1}\right)}\right) .
$$

Далее возьмем функцию $\alpha_{2}(x) \in C_{0}^{\infty}\left(\mathscr{Y}_{1}\right)$, обладаюшую следуюшими свойствами. Пусть $D_{2}=\left\{x: \alpha_{2}(x)=1\right\}$, причем сушествует открытая область $B_{2}$, $B_{2} \subset D_{2}, \operatorname{supp} \psi(x) \subset B_{2}, \operatorname{supp} \alpha_{2}(x) \subset B_{1}$. Тогда $\alpha_{2}(x) \cdot \alpha_{1}(x)=\alpha_{2}(x)$ и сушествует такое $h_{1}>0$, что при $|h|<h_{1}$ будет выполняться

$$
\left(\prod_{h}^{*} \alpha_{2}(x)\right)\left(\prod_{h}^{*} \alpha_{1}(x)\right)=\prod_{h}^{*} \alpha_{2}(x) .
$$

Рассмотрим выражение

$$
\begin{aligned}
T_{h}^{-1}[ & \left.\left(\prod_{h}^{*} \alpha_{2}(x)\right) A_{h} a(x, h)\right] \\
= & T_{h}^{-1}\left[\left(\prod_{h}^{*} \alpha_{2}(x)\right) T_{h} a(x, h)\right]+T_{h}^{-1}\left[\left(\prod_{h}^{*} \alpha_{2}(x)\right) F_{h} a(x, h)\right] \\
= & \left(\prod_{h}^{*} \alpha_{2}(x)\right) a(x, h)+T_{h}^{-1}\left[( \prod _ { h } ^ { * } \alpha _ { 2 } ( x ) ) T _ { h } \left(\left(\prod_{h}^{*} \alpha_{1}(x)\right) a(x, h)\right.\right. \\
& \left.\left.-T_{h}\left(\prod_{h}^{*} \alpha_{1}(x)\right)\left(\prod_{h}^{*} \alpha_{2}(x)\right) a(x, h)\right)\right] \\
& +T_{h}^{-1}\left[\left(\prod_{h}^{*} \alpha_{2}(x)\right) T_{h}\left(\prod_{h}^{*}\left(\Theta(x)-\alpha_{1}(x)\right)\right)\right] \\
& +T_{h}^{-1}\left[\left(\prod_{h}^{*} \alpha_{2}(x)\right) F_{h} a(x, h)\right] \\
= & T_{h}^{-1}\left[\left(\prod_{h}^{*} \alpha_{2}(x)\right) \prod_{h} f(x)\right] .
\end{aligned}
$$

Соотношение (2.18) перепишем в виде

$$
\sum_{i=1}^{4} I_{i}(h)=I_{5}(h) .
$$

Функция $\Theta(x) \in C_{0}^{\infty}\left(\mathbb{R}_{n}\right), \Theta(x)=1$ при $x \in \Omega$. Носители функций $\prod_{h}^{*} \alpha_{2}(x)$ и $\prod_{h}^{*}\left(\Theta(x)-\alpha_{1}(x)\right)$ не пересекаются для всех $h,|h|<h_{2}$. Тогда согласно свойству псевдолокальности $\forall s \exists h_{3}>0$ такое, что при $|h|<h_{4}, h_{4}=\min \left\{h_{1}, h_{2}, h_{3}\right\}$, будет вьполняться оценка

$$
\begin{aligned}
& \left\|T_{h}^{-1}\left[\left(\prod_{h}^{*} \alpha_{2}(x)\right) T_{h}\left(\prod_{h}^{*}\left(\Theta(x)-\alpha_{1}(x)\right) a(x, h)\right)\right]\right\|_{\stackrel{\circ}{\circ\left(s, h, \Omega_{h}\right)}} \\
& \quad \leqslant C_{r s}\|f(x)\|_{L_{2}(\Omega)} .
\end{aligned}
$$


Функция $g_{11}(x, h)=T_{h}^{-1}\left[\left(\prod_{h}^{*} \alpha_{2}(x)\right) \prod_{h} f(x)\right] \in M(\lambda+2 r, h)$, причем выполняется оценка

$$
\left\|g_{11}(x, h)\right\|_{M(\lambda+2 r, h)} \leqslant C_{1 \lambda r}\|f(x)\|_{H_{\lambda}\left(\mathscr{Y}_{1}\right)} .
$$

Учитьвая неравенство (2.17) и предложение 1.3 , получаем

$$
\begin{aligned}
\| T_{h}^{-1} & {\left[\left(\prod_{h}^{*} \alpha_{2}(x)\right) T_{h}\left(\left(\prod_{h}^{*} \alpha_{1}(x)\right) a(x, h)\right)\right.} \\
& \left.\quad-T_{h}\left(\left(\prod_{h}^{*} \alpha_{2}(x)\right)\left(\prod_{h}^{*} \alpha_{1}(x)\right) a(x, h)\right)\right] \|_{M\left(s_{1}+1, h\right)} \\
\leqslant & C_{2 \lambda r}\left(\|f(x)\|_{L_{2}(\Omega)}+\|f\|_{H_{\lambda}\left(\mathscr{Y}_{1}\right)}\right) .
\end{aligned}
$$

Для слагаемого $I_{3}(h)$ выполняется оценка $(2.15)$. Слагаемое $I_{4}(h)$ можно записать в виде

$$
\begin{aligned}
T_{h}^{-1}[ & \left.\left(\prod_{h}^{*} \alpha_{2}(x)\right) F_{h} a(x, h)\right] \\
= & T_{h}^{-1} F_{h}\left(\left(\prod_{h}^{*} \alpha_{2}(x)\right) a(x, h)\right) \\
& +T_{h}^{-1}\left[( \prod _ { h } ^ { * } \alpha _ { 2 } ( x ) ) F _ { h } \left(\left(\prod_{h}^{*} \alpha_{1}(x)\right) a(x, h)\right.\right. \\
& \left.\left.-F_{h}\left(\prod_{h}^{*} \alpha_{1}(x)\right)\left(\prod_{h}^{*} \alpha_{2}(x)\right) a(x, h)\right)\right] \\
& +T_{h}^{-1}\left[\left(\prod_{h}^{*} \alpha_{2}(x)\right) F_{h}\left(\left(\prod_{h}^{*}\left(\Theta(x)-\alpha_{1}(x)\right)\right) a(x, h)\right)\right] \\
= & \sum_{i=1}^{3} f_{i}(x, h) .
\end{aligned}
$$

Выше было доказано, что если

$$
\left(\prod_{h}^{*} \alpha_{1}(x)\right) a(x, h) \in \stackrel{\circ}{M}\left(s_{1}, h, \mathscr{Y}_{1 h}\right),
$$

где $s_{1}=\min (r+\varepsilon, 2 r+\lambda)$, то для функции

$$
\left(\prod_{h}^{*} \alpha_{2}(x)\right) a(x, h) \in \stackrel{\circ}{M}\left(s_{1}, h, \mathscr{Y}_{1 h}\right)
$$

будет равномерно выполняться оценка $(2.17)$ при $|h|<h_{4}$. Символ оператора $F_{h}$ принадлежит классу $S_{h(2 r-\varepsilon)}^{\infty}$, поэтому из последних замечаний вытекает оценка

$$
\left\|f_{1}(x, h)\right\|_{M\left(s_{1}+\varepsilon, h\right)} \leqslant C_{3 \lambda r}\left(\|f(x)\|_{L_{2}(\Omega)}+\|f(x)\|_{H_{\lambda}\left(\mathscr{Y}_{1}\right)}\right)
$$

где $C_{3 \lambda r}$ не зависит от $h=\left(h_{1}, \ldots, h_{n}\right)$.

Носители функций $\prod_{h}^{*} \alpha_{2}(x)$ и $\prod_{h}^{*}\left(\Theta(x)-\alpha_{1}(x)\right)$ не пересекаются при $|h|<h_{4}$. Тогда согласно свойству псевдолокальности для любого $k$ существует $h_{5}>0$, $h_{5}<h_{4}$, такое, что при $|h|<h_{5}$ будет выполняться оценка

$$
\left\|f_{3}(x, h)\right\|_{M(k, h)} \leqslant C_{k r}^{(1)}\left(\|f(x)\|_{L_{2}(\Omega)}+\|f(x)\|_{H_{\lambda}\left(\mathscr{Y}_{1}\right)}\right) .
$$


Из предложения 1.3 и неравенства (2.17) следует

$$
\left\|f_{2}(x, h)\right\|_{M\left(s_{1}+\varepsilon, h\right)} \leqslant C_{4}\left(\|f(x)\|_{L_{2}(\Omega)}+\|f(x)\|_{H_{\lambda}\left(\mathscr{Y}_{1}\right)}\right) .
$$

Из оценок (2.24)-(2.26) вытекает неравенство

$$
\left\|T_{h}^{-1}\left[\left(\prod_{h}^{*} \alpha_{2}(x)\right) F_{h} a(x, h)\right]\right\|_{M\left(s_{1}+\varepsilon, h\right)} \leqslant C_{5}\left(\|f(x)\|_{L_{2}(\Omega)}+\|f(x)\|_{H_{\lambda}\left(\mathscr{Y}_{1}\right)}\right),
$$

где $C_{5}$ не зависит от $h=\left(h_{1}, \ldots, h_{n}\right)$.

Из оценок (2.18)-(2.22) и (2.27) следует оценка

$$
\left\|\left(\prod_{h}^{*} \alpha_{2}(x)\right) a(x, h)\right\|_{M\left(s_{2}, h, \Omega_{1 h}\right)} \leqslant C_{6}\left(\|f(x)\|_{L_{2}(\Omega)}+\|f(x)\|_{H_{\lambda}\left(\mathscr{Y}_{1}\right)}\right),
$$

где $s_{2}=\min \left(s_{1}+\varepsilon, 2 r+\lambda\right)$.

Рассуждая далее аналогично, получаем утверждение теоремы 2.2 .

ЗАмечАниЕ 2.1. Как видно из доказательства теоремы 2.2, утверждение теоремы останется справедливым, если для оператора $F_{h}$ будут вьполняться условия:

1) семейство операторов $F_{h}$ равномерно ограниченно отображает $M(s, h)$ в $M(s-2 r+\varepsilon, h)$ для любого $s$;

2) для семейства операторов $F_{h}$ выполняется оценка

$$
\begin{aligned}
& \left\|\left(\prod_{h}^{*} \alpha(x)\right) F_{h} a(x, h)-F_{h}\left(\left(\prod_{h}^{*} \alpha(x)\right) a(x, h)\right)\right\|_{M(s-2 r+1+\varepsilon, h)} \\
& \quad \leqslant C_{s}\|a(x, h)\|_{M(s, h)},
\end{aligned}
$$

где $C_{s}$ не зависит от $h=\left(h_{1}, \ldots, h_{n}\right), \alpha(x) \in C_{0}^{\infty}\left(\mathbb{R}_{n}\right), s$ произвольно;

3 ) сушествует $h_{0}>0$ такое, что при $|h|<h_{0}$ выполняется оценка (свойство псевдолокальности)

$$
\left\|\left(\prod_{h}^{*} \alpha(x)\right) F_{h}\left(\left(\prod_{h}^{*} \beta(x)\right) a(x, h)\right)\right\|_{M(s, h)} \leqslant C_{s r}\|a(x, h)\|_{M(r, h)},
$$

где $C_{s r}$ не зависит от $h=\left(h_{1}, \ldots, h_{n}\right), \alpha(x), \beta(x) \in C_{0}^{\infty}\left(\mathbb{R}_{n}\right)$, носители функций $\alpha(x)$ и $\beta(x)$ не пересекаются, $a(x, h) \in M(r, h), r$ и $s$ произвольны.

ПримеР 2.1. Рассмотрим семейство операторов, определенное соотношением

$$
F_{h} u(x, h)=\left(\prod_{h}^{*} b(x)\right) T_{\text {пр }} u(x, h)+u(x, h)\left(\prod_{h}^{*} \lambda(x)\right),
$$

где $b(x), \lambda(x) \in C_{0}^{\infty}\left(\mathbb{R}_{1}\right), u(x, h) \in M(r, h), T_{\text {пр }}$ - правьй разностный оператор первого порядка.

Семейство операторов $F_{h}$ равномерно ограниченно отображает пространства $M(s, h)$ в $M(s-1, h)$. Очевидно, что выполняется неравенство (2.29). Проверим свойство 2). Действительно, имеет место соотношение

$$
\begin{gathered}
\pi\left\{\left(\prod_{h}^{*} \alpha(x)\right) F_{h} a(x, h)-F_{h}\left(\left(\prod_{h}^{*} \alpha(x)\right) a(x, h)\right)\right\} \\
=-b(k h) u((k+1) h) \frac{\alpha((k+1) h)-u(k h)}{h},
\end{gathered}
$$

где $\alpha(x) \in C_{0}^{\infty}\left(\mathbb{R}_{1}\right), u((k+1) h)=u((k+1) h, h), \pi$ - оператор, заданный определением 1.2.

Из предложения 1.1 и (2.30) следует соотношение (2.29). Отсюда вытекает, что при $r=1$ для семейства операторов $F_{h}$ справедливо замечание 2.1 . 
ПримеР 2.2. Рассмотрим разностный оператор $T_{h}=\left(\Delta_{p}\right)^{m}+(-1)^{m}$, где $\Delta_{p}-$ разностный оператор Лапласа. Сеточный символ оператора $T_{h}$ имеет вид (см. [1])

$$
\widehat{T}_{c}(\varphi, h)=(-1)^{m} 4^{m}\left[\sum_{i=1}^{n} \frac{1}{h_{i}^{2}} \sin ^{2} \frac{\varphi_{i}}{2}\right]^{m}+(-1)^{m} .
$$

Из (2.31) следует соотношение для ступенчатого символа $\widehat{T}_{h}(t, h)$

$$
\widehat{T}_{h}(t, h)=(-1)^{m} 4^{m}\left[\sum_{i=1}^{n} \frac{1}{h_{i}^{2}} \sin ^{2} \frac{t_{i} h_{i}}{2}\right]^{m}+(-1)^{m}
$$

Используя (2.32), получаем оценку

$$
C_{1}\left(1+\sum_{i=1}^{n} \frac{1}{h_{i}}\left|\sin \frac{t_{i} h_{i}}{2}\right|\right)^{2 m} \leqslant\left|\widehat{T}_{h}(t, h)\right| \leqslant C_{2}\left(1+\sum_{i=1}^{n} \frac{1}{h_{i}}\left|\sin \frac{t_{i} h_{i}}{2}\right|\right)^{2 m} .
$$

Из (2.33) следует, что $\widehat{T}_{h}(t, h) \in S_{h(2 m)}$. Непосредственно проверяется, что $\widehat{T}_{h}(t, h) \in S_{h(2 m)}^{\infty}$.

Рассмотрим теперь задачу Пуассона с однородными условиями Дирихле в области $\mathscr{Y ~ , ~ у д о в л е т в о р я ю щ е и ̆ ~ у с л о в и я м ~ т е о р е м ы ~ 2 . 1 : ~}$

$$
\begin{aligned}
& \Delta^{m} u+(-1)^{m} u=f(x), \quad x \in \mathscr{Y}, \\
& \left.u\right|_{\Gamma}=0,
\end{aligned}
$$

где $f(x) \in L_{2}(\mathscr{Y}), f(x) \in C^{\infty}\left(\mathscr{Y}_{1}\right), \overline{\mathscr{Y}}_{1} \subset \mathscr{Y}, \Gamma-$ граница области $\mathscr{Y ~}$

Приближенное решение $u(x, h)$ задачи (2.34) будем искать из системы

$$
p_{h}\left(\Delta_{p}^{m} u(x, h)+(-1)^{m} u(x, h)\right)=p_{h}\left(\prod_{h} f(x)\right),
$$

где $p_{h}$ - оператор сужения на область $\mathscr{Y}_{h}$ (см. [2]).

Из результатов [2] следует, в частности, что решение $u(x) \in \stackrel{\circ}{H}_{r}(\mathscr{Y})$ сушествует и приближенные решения $u(x, h)$ сходятся к $u(x)$ по норме $\stackrel{\circ}{H}_{r}(\mathscr{Y}), 0<r<1 / 2$. Из следствия 2.2 вытекает, что разностные отношения $u(x, h)$ любого порядка сходятся равномерно в любой области $\mathscr{Y}_{2}$ такой, что $\overline{\mathscr{Y}}_{2} \subset \mathscr{Y}_{1}$.

ПримеР 2.3. Рассмотрим задачу Пуассона

$$
\begin{aligned}
& A u=f(x), \quad x \in \mathscr{Y}, \\
& \left.u\right|_{\Gamma}=0,
\end{aligned}
$$

где $A u=\Delta u+F u=\Delta u+\sum_{i=1}^{n} b_{i}(x) \frac{\partial u}{\partial x_{i}}+\lambda(x) u(x), \Delta$ - оператор Лапласа, область $\mathscr{Y}$ и правая часть функции $f(x)$ удовлетворяют тем же условиям, что и в примере $2.2, b_{i}(x), \lambda(x) \in C^{\infty}\left(\mathbb{R}_{n}\right)$.

Приближенное решение $u(x, h)$ задачи $(2.36)$ будем искать из системы

$$
p_{h} A_{h} u(x, h)=p_{h}\left(\prod_{h} f(x)\right)
$$

где $A_{h}=T_{h}+F_{h}, T_{h}=\Delta_{p}, F_{h}=\sum_{i=1}^{n}\left(\prod_{h}^{*} b_{i}(x)\right) T_{\text {пр }}(i) u(x, h)+\left(\prod_{h}^{*} \lambda(x)\right) u(x, h)$, здесь $T_{\text {пр }}(i)$ означает, что правая разностная производная берется по $i$-й переменной. Из примера 2.1 следует, что для оператора $A_{h}$ справедливо замечание 2.1. Поэтому для задачи (2.36) справедливы все утверждения примера 2.2 (см. [2]). 
ПримеР 2.4. Рассмотрим задачу Пуассона

$$
\begin{aligned}
& \Delta^{m} u=f(x), \quad x \in \mathscr{Y}, \\
& \left.u\right|_{\Gamma}=0 .
\end{aligned}
$$

Область $\mathscr{Y}$ и функция $f(x)$ удовлетворяют тем же условиям, что и в примере 2.2 . Приближенное решение $u(x, h)$ задачи $(2.38)$ будем искать из системы

$$
p_{h} \Delta_{p}^{m} u(x, h)=p_{h}\left(\prod_{h} f(x)\right) .
$$

Оператор $\Delta_{p}^{m}$ можно представить в виде

$$
\Delta_{p}^{m}=\left(\Delta_{p}^{m}+(-1)^{m}\right)+(-1)^{m+1}=T_{h}+F_{h}=A_{h} .
$$

Для оператора $A_{h}$ выполняются все условия теоремы 2.2. Поэтому для задач (2.38) и (2.39) справедливы все утверждения примера 2.2 .

ПримеР 2.5. Рассмотрим одномерный оператор Прандтля

$$
A u=\int_{-\infty}^{\infty} \frac{u\left(x_{0}\right) d x_{0}}{\left(x-x_{0}\right)^{2}},
$$

где $u\left(x_{0}\right) \in H_{r}(\mathbb{R})$.

Ось $O X$ разобьем с шагом $h$ точками $x_{i}=h(i-1 / 2), i \in \mathbb{Z}$. Тогда вся числовая ось разобьется на интервалы $D(k, h)=((k-1 / 2) h,(k+1 / 2) h)$. Определим числа $\gamma_{i}, i \in \mathbb{Z}$, соотношением

$$
\gamma_{i}=\int_{D(i, h)} \frac{d x_{0}}{x_{0}^{2}}=\frac{1}{h} \cdot \frac{1}{i^{2}-1 / 4} .
$$

В (2.41) при $i=0$ интеграл понимается по Адамару.

Дискретный оператор Прандтля определим формулой

$$
\pi A_{h} u(x, h)=\sum_{i=-\infty}^{\infty} \gamma_{k-i} a(i)
$$

где $a(i)=\pi u(x, h)$.

Оператор $A_{h}$ можно представить в виде псевдоразностного оператора

$$
\pi A_{h} u(x, h)=\frac{1}{2 \pi} \int_{-\pi}^{\pi} \widehat{A}_{c}(\varphi, h) \widehat{a}_{c}(\varphi) e^{i k \varphi} d \varphi,
$$

где $\widehat{a}_{c}(\varphi)$ - сеточное преобразование Фурье.

Для сеточного и ступенчатого символов п.р.о. имеют место соотношения

$$
\widehat{A}_{c}(\varphi, h)=\sum_{k=-\infty}^{\infty} \gamma_{k} e^{i k \varphi}, \quad \widehat{A}_{h}(t, h)=\widehat{A}_{c}(t h, h)=\sum_{k=-\infty}^{\infty} \gamma_{k} e^{i k h t} .
$$


Построим новый оператор $B_{h}$ следующим образом: возьмем четную функцию $\chi(x, a) \in C_{0}^{\infty}(\mathbb{R})(a$ - параметр) такую, что $0 \leqslant \chi(x, a) \leqslant 1, \chi(x, a)=1$ при $-a \leqslant x \leqslant a$, и определим оператор $B_{h}$ :

$$
\pi B_{h} u(x, h)=\sum_{i=-\infty}^{\infty} \beta_{k-i} a_{i}
$$

где $\beta_{k}=\gamma_{k} \chi(k, h)$.

Ступенчатый символ $\widehat{B}_{h}(t, h)$ п.р.о. $B_{h}$ имеет вид

$$
\widehat{B}_{h}(t, h)=\sum_{k=-\infty}^{\infty} \beta_{k} e^{i k h t}=-4 \sum_{k=-\infty}^{\infty} \beta_{k} \sin ^{2} \frac{k h t}{2}+O(h),
$$

где $O(h)=\beta_{0}+2 \sum_{k=1}^{\infty} \beta_{k}$. Из конструкции функции $\chi(x, a)$ следует неравенство

$$
C_{1}(a) \leqslant|O(h)| \leqslant C_{2}(a)
$$

где $C_{i}(a) \geqslant 0$ не зависят от $h$.

В работах [1], [2] доказано неравенство при $-\pi / h \leqslant t \leqslant \pi / h$

$$
\frac{C_{3}}{2}\left|\sin \frac{t h}{2}\right| \leqslant 4 \sum_{k=1}^{\infty} \beta_{k} \sin ^{2} \frac{k h t}{2} \leqslant \frac{C_{4}}{2}\left|\sin \frac{t h}{2}\right|
$$

Из неравенств (2.45) и (2.46) следует, что оператор $B_{h}$ является сильно эллиптическим и принадлежит классу $S_{h 1}$.

Докажем, что $\widehat{B}_{h}(t, h) \in S_{h 1}^{\infty}$. Рассмотрим выражение

$$
\frac{d^{n} \widehat{B}_{h}(t, h)}{d t^{n}}=\sum_{k=-\infty}^{\infty} i^{n} \chi(k h) \gamma_{k}(k h)^{n} e^{i k h t} .
$$

Для преобразования этого выражения используем тождество

$$
\gamma_{k} h k^{n}=\frac{k^{n}}{k^{2}-1 / 4}=\frac{a_{n} k+b_{n}}{k^{2}-1 / 4}+P_{n-2}(k),
$$

где $P_{n-2}(k)$ - многочлен степени $n-2$ относительно $k$ :

$$
\begin{aligned}
\frac{d^{n} \widehat{B}_{h}(t, h)}{d t^{n}}= & i^{n} b_{n} \sum_{k=-\infty}^{\infty} \chi(k h) \gamma_{k} e^{i k h t} h^{n} \\
& +i^{n} a_{n} \sum_{k=-\infty}^{\infty} \chi(k h) \frac{k}{k^{2}-1 / 4} h^{n-1} e^{i k h t} \\
& +i^{n} \sum_{k=-\infty}^{\infty} \chi(k h) P_{n-2}(k) h^{n-2} e^{i k h t} \\
= & \sum_{j=1}^{3} \widehat{B}_{j n}(t, h) .
\end{aligned}
$$


Фиксируем некоторое $h_{0}>0$, тогда при $|h|<h_{0}$ имеет место оценка

$$
\left|\widehat{B}_{1 n}(t, h)\right| \leqslant k_{1 n}\left(1+\frac{1}{h}\left|\sin \frac{t h}{2}\right|\right)^{1-n}
$$

где $t \in[-\pi / 4, \pi / 4]$.

Ввиду равенства

$$
P_{n-2}(k) h^{n-2}=C_{0}(k h)^{n-2}+C_{1} h(k h)^{n-2}+\cdots+C_{n-3}(k h) h^{n-3}+C_{n-2} h^{n-2}
$$

к $\widehat{B}_{3 n}(t, h)$ применимо предложение 1.1. Поэтому вьполняется неравенство

$$
\left|\widehat{B}_{3 n}(t, h)\right| \leqslant k_{2 n}\left(1+\frac{1}{h}\left|\sin \frac{t h}{2}\right|\right)^{1-n} .
$$

Преобразуем слагаемое $\widehat{B}_{2 n}(t, h)$ :

$$
\begin{aligned}
\widehat{B}_{2 n}(t, h) & =2 i^{n} a_{n} \sum_{k=1}^{\infty} \frac{\chi(k h)}{k+1 / 2}(\sin t k h) h^{n-1}+i^{n} a_{n} \sum_{k=1}^{\infty} \frac{\chi(k h)}{k^{2}-1 / 4}(\sin t k h) h^{n-1} \\
& =I_{1}(h)+I_{2}(h)
\end{aligned}
$$

Очевидно, что при $t \in[-\pi / 4, \pi / 4)$ справедливо неравенство

$$
\left|I_{2}(h)\right| \leqslant k_{3 n}\left(1+\frac{1}{h}\left|\sin \frac{t h}{2}\right|\right)^{1-n} .
$$

Докажем, что имеет место оценка

$$
\left|\sum_{k=1}^{\infty} \frac{\chi(k h)}{k+1 / 2} \sin t k h\right| \leqslant C_{0}
$$

Для величин $\alpha_{k}=\frac{\chi(k h)}{k+1 / 2}$ выполняются оценки

$$
\left|\alpha_{k}\right| \leqslant \frac{A_{1}}{k+1 / 2}, \quad\left|\alpha_{k}-\alpha_{k+1}\right| \leqslant \frac{A_{2}}{k^{2}}+\frac{A_{3} h}{k},
$$

где $A_{i}, i=1,2,3$, не зависят от $h$.

Так как функция $\chi(x, a)$ финитна, то существует такой отрезок $[-b, b]$, для которого $\operatorname{supp} \chi(x, a) \subset[-b, b], b=p h, p \in \mathbb{N}$. Отсюда следует, что для всех $1 \leqslant k \leqslant p$ имеет место неравенство $1 / k \geqslant 1 / p=h / b=D_{0} h$.

Кроме того, очевидно соотношение

$$
\sum_{k=1}^{\infty} \alpha_{k} \sin t k h=\sum_{k=p}^{\infty} \alpha_{k} \sin t k h
$$


Из последних замечаний и (2.55) следует неравенство

$$
\left|\alpha_{k}-\alpha_{k+1}\right| \leqslant \frac{A_{3}}{k^{2}}
$$

где $A_{3}$ не зависит от $h$.

При условиях (2.55), (2.56) в [2] доказано неравенство (2.54). Из (2.52) и (2.54) вытекает оценка

$$
\left|\widehat{B}_{2 n}(t, h)\right| \leqslant k_{2 n}\left(1+\frac{1}{h}\left|\sin \frac{t h}{2}\right|\right)^{1-n} .
$$

Из (2.49)-(2.51), (2.57) следует неравенство

$$
\left|\frac{d^{n} \widehat{B}_{h}(t, h)}{d t^{n}}\right| \leqslant D_{n}\left(1+\frac{1}{h}\left|\sin \frac{t h}{2}\right|\right)^{1-n}
$$

Итак, символ $\widehat{B}_{h}(t, h) \in S_{h 1}^{\infty}$.

Рассмотрим уравнение

$$
\int_{c}^{d} \frac{u\left(x_{0}\right) d x_{0}}{\left(x-x_{0}\right)^{2}}=f(x)
$$

где $f(x) \in L_{2}[c, d], f(x) \subset C^{\infty}\left(\mathscr{Y}_{1}\right), \mathscr{Y}_{1}$ - открытая область, $\overline{\mathscr{Y}}_{1} \subset[c, d], u\left(x_{0}\right) \in$ $\stackrel{\circ}{H}_{1 / 2}[c, d]$.

Всегда можно подобрать такую функцию $\chi(x, a)$, что уравнение $(2.58)$ и уравнение

$$
\int_{c}^{d} g\left(x-x_{0}\right) u(x) d x_{0}=f(x)
$$

будут эквивалентны.

Кроме того, будут совпадать системы линейных алгебраических уравнений (С.Л.А.У.) для нахождения приближенных решений уравнений (2.58) и (2.59) соответственно:

$$
\begin{aligned}
& \sum_{i \in \Lambda(h)} \gamma_{i j} u(i h, h)=f_{h}\left(x_{j}\right), \quad j \in \Lambda(h), \\
& \sum_{i \in \Lambda(h)} \beta_{i j} V(i h, h)=f_{h}\left(x_{j}\right), \quad j \in \Lambda(h),
\end{aligned}
$$

где $\gamma_{i j}=\gamma_{j-i}, \beta_{i j}=\beta_{j-i}, \Lambda(h)$ - множество индексов $j$ таких, что $((j-1 / 2) h$, $(j+1 / 2) h) \subset(c, d), f_{h}\left(x_{j}\right)$ - значение интегрального проектора $\prod_{h} f(x)$ на интервале $((j-1 / 2) h,(j+1 / 2) h) \subset(c, d)$.

Псевдоразностный оператор $B_{h}$ соответствующей С.Л.А.У. (2.61) принадлежит классу $S_{h 1}^{\infty}$ и является сильно эллиптическим. Отсюда и из результатов [2] вытекает, что для (2.58) и (2.60) справедливы все утверждения примера 2.2. 


\section{Список литературы}

1. Лифанов И. К., Полтавский Л. Н. Пространства дробных отношений, дискретные операторы и их приложения. I // Матем. сб. 1999. Т. 190. №9. С. 41-98.

2. Лифанов И. К., Полтавский Л. Н. Пространства дробных отношений, дискретные операторы и их приложения. II // Матем. сб. 1999. Т. 190. № 11. С. 67-134.

3. Зигмунд А. Тригонометрические ряды. Т. 1, 2. М.: ИЛ, 1962.

Военный авиационный технический университет им. Н.Е. Жуковского,

г. Москва
Поступила в редакцию

11.02 .2001 